LEDO MAZZEI MASSONI NETO

\title{
Avaliação da pressão arterial antes e após paratireoidectomia por hiperparatireoidismo primário
}

Tese apresentada à Faculdade de Medicina da Universidade de São Paulo para obtenção do título de Doutor em Ciências

Programa de Clínica Cirúrgica

Orientador: Prof. Dr. Fábio Luiz de Menezes Montenegro

VERSÃO CORRIGIDA

Resolução CoPGr 6018/11, de 1/11/2011

São Paulo

2018 


\section{Dados Internacionais de Catalogação na Publicação (CIP)}

\section{Preparada pela Biblioteca da}

Faculdade de Medicina da Universidade de São Paulo

Creprodução autorizada pelo autor

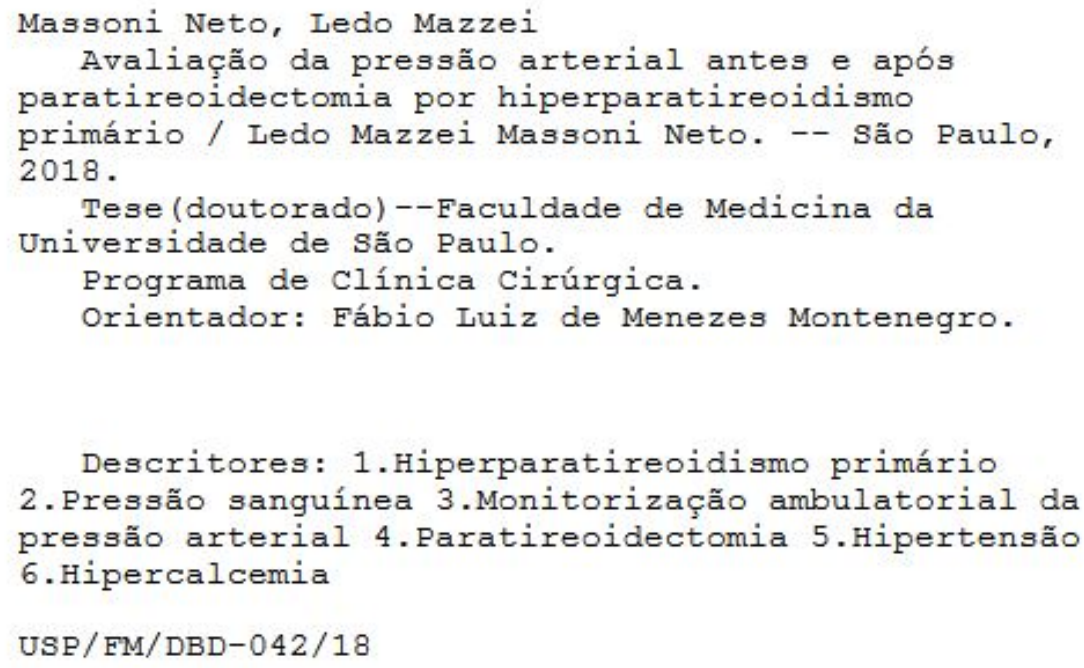

Responsável: Kátia Maria Bruno Ferreira - CRB-8/6008 


\section{Dedicatória}

Aos meus pais, Ledo e Eugênia, que me ensinaram os verdadeiros valores da vida

À minha irmã Nathália, e meu sobrinho João Pedro, presentes apesar de longe

Aos meus filhos Lucas e Pedro, maior realização da minha vida e motivo pra tentar sempre ser um homem melhor

À minha amada esposa Viviane, pelo carinho, incentivo e por ser o meu norte quando me perco no turbilhão do dia a dia 


\section{Agradecimentos}

Ao Prof Dr Fabio Luiz de Menezes Montenegro, orientador desta tese, pelo estímulo constante e paciente, exemplo de dedicação, integridade e generosidade,

Ao Dr Climério Pereira do Nascimento, pela amizade dentro e fora de campo, exemplo de resiliência e bom humor inabaláveis

Ao Prof Dr Sérgio Samir Arap, e à Dra Marília D'Elboux Guimarães Brescia, pelo aprendizado da especialidade na residência e convivio enriquecedor no Grupo de Paratireoide

Ao Dr Caio Tosato Caliseo, amigo e companheiro no primeiro trabalho fora do $\mathrm{HC}$, o IBCC. Agradeço pela preceptoria e coleguismo

Ao Prof Dr Lenine Garcia Brandão, que após a residência me ofereceu oportunidade de trabalhar no IBCC, hospital onde aprendi e aprendo muito até hoje com os colegas da especialidade e residentes de cirurgia geral

À Dra Jossi Ledo Kanda, que ofereceu ao recém egresso da residência a oportunidade de participar de um serviço de Cirurgia de Cabeça e Pescoço como assistente, experiência fundamental na minha formação

Aos médicos assistentes da Disciplina de Cirurgia de Cabeça e Pescoço do HC-FMUSP que participaram da minha formação na especialidade

Ao Prof Dr Décio Mion Jr, da Nefrologia do HC-FMUSP, que permitiu que realizássemos as MAPAS do presente estudo, além de discussões desde a concepção do trabalho 
Aos médicos do serviço de Endocinologia do HC-FMUSP, Dr Pedro Henrique Silveira Corrêa e Dra Regina Martsunaga Martin, que tanto ajudaram, permitindo que seus pacientes participassem do presente estudo, e pelas ricas discussões

Aos funcionários do HC-FMUSP, que sempre foram solícitos e me ajudaram no cuidado aos pacientes, no centro cirúrgico, setores de internação e ambulatórios, e em especial do setor de MAPA da nefrologia, na figura da enfermeira Jovita

À Sra Eliane Falconi Mônico Gazetto, a quem conheço desde que fui aluno de iniciação científica, que sempre ajudou com o cumprimento de prazos e formalidades

Aos pacientes que permitiram que o aluno, o médico e o cirurgião pudessem aprender com seu sofrimento. Em especial aos pacientes que aceitaram participar do estudo dessa tese, que aceitaram incômodos adicionais ao seu tratamento sem nenhum benefício direto, apenas a promessa de contribuir para 0 entendimento da doença que portavam, para possível benefício de futuros pacientes.

À CAPES pelo auxílio na forma de Bolsa,do Programa de Demanda Social que contribuiu para que pudesse me dedicar ao presente trabalho

A todos que colaboraram com este trabalho mas não estiveram aqui relacionados, minhas desculpas e minha gratidão. 
"Aqueles que passam por nós, não vão sós, não nos deixam sós. Deixam um pouco de si, levam um pouco de nós.

- Antoine de Saint-Exupéry 
Autorizo a reprodução e divulgação total ou parcial deste trabalho por qualquer meio convencional ou eletrônico, para fins de estudo e pesquisa, desde que citada a fonte 


\section{Normatização adotada}

Esta tese está de acordo com as seguintes normas, em vigor no momento desta publicação:

Referências: adaptado de International Committee of Medical Journals Editors (Vancouver).

Universidade de São Paulo. Faculdade de Medicina. Divisão de Biblioteca e Documentação. Guia de apresentação de dissertações, teses e monografias. Elaborado por Anneliese Carneiro da Cunha, Maria Julia de A. L. Freddi, Maria F. Crestana, Marinalva de Souza Aragão, Suely Campos Cardoso, Valéria Vilhena. 3a ed. São Paulo: Divisão de Biblioteca e Documentação; 2011.

Abreviaturas dos títulos dos periódicos: de acordo com List of Journals Indexed in Index Medicus. 


\section{Sumário}

Lista de abreviaturas e siglas

Lista de símbolos

Lista de tabelas

Lista de figuras

Normatização adotada

Sumário

Resumo

Summary / Abstract

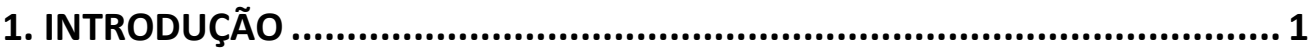

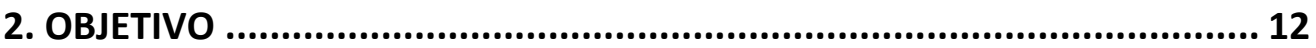

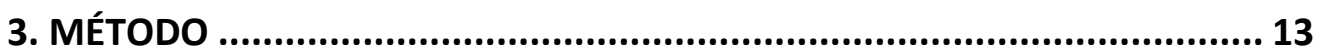

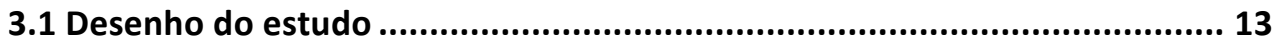

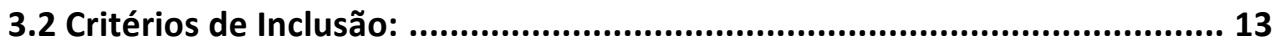

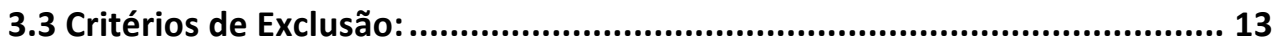

3.4 Exames laboratoriais:................................................................................ 14

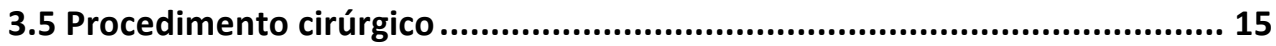

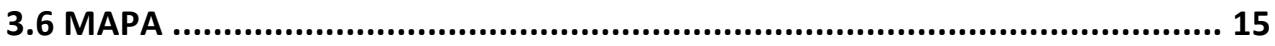

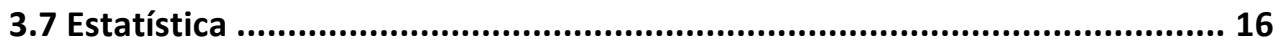

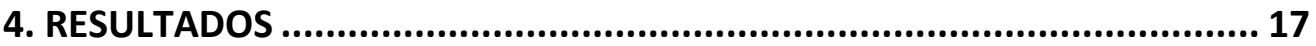

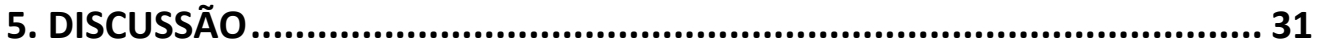

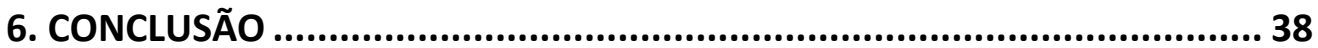

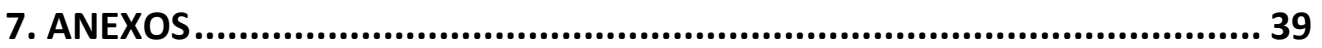

Anexo A - Termo de Consentimento Livre e Esclarecido ................................ 39

Anexo B - Planejamento das MAPAS ......................................................... 42

Anexo C - Aprovação pelo Comitê de Ética em Pesquisa ................................. 43

Anexo D - Alteração da finalidade acadêmica para doutorado ........................ 44

Anexo E - Dados demográficos e bioquímicos dos 22 pacientes incluídos....... 45

Anexo F - Descenso noturno da pressão arterial sistêmica dos 22 casos (\%) ... 46

8. REFERÊNCIAS BIBLIOGRÁFICAS ..................................................... 47 


\section{LISTA DE ABREVIATURAS E SIGLAS}

$\begin{array}{ll}\text { 3PO } & \text { terceiro pós-operatório } \\ \text { ANOVA } & \text { Análise de variância } \\ \text { CAAE } & \text { Certificado de Apresentação para Avaliação Ética } \\ \text { Cai } & \text { Cálcio ionizado } \\ \text { CaT } & \text { Cálcio total } \\ \text { EPM } & \text { Erro padrão da media } \\ \text { et al } & \text { e outros } \\ \text { HCFMUSP } & \text { Hospital das Clínicas da Faculdade de Medicina da Universidade de São } \\ & \text { Paulo } \\ \text { HPTP } & \text { Hiperparatireoidismo primário } \\ \text { MAPA } & \text { Monitorização ambulatorial da pressão arterial } \\ \text { n } & \text { Número de pacientes } \\ \text { p } & \text { Nível descritivo calculado } \\ \text { P } & \text { Fósforo } \\ \text { PAD } & \text { Pressão arterial diastólica } \\ \text { PAS } & \text { Pressão arterial sistólica } \\ \text { PRE } & \text { Pré-operatório } \\ \text { PTH } & \text { Paratormônio } \\ \text { Q1-Q3 } & \text { Intervalo interquartis } \\ \text { RR } & \text { Risco relativo }\end{array}$




\section{LISTA DE SÍMBOLOS}

$\begin{array}{ll}\mathrm{dL} & \text { Decilitro } \\ \mathrm{g} & \text { Grama } \\ \mathrm{L} & \text { Litro } \\ \mathrm{mcg} & \text { Micrograma } \\ \mathrm{mg} & \text { Miligrama } \\ \mathrm{mL} & \text { Mililitro } \\ \mathrm{mmHg} & \text { milímetros de mercúrio } \\ \mathrm{pg} & \text { Picograma } \\ \pm & \text { mais ou menos } \\ < & \text { menor que } \\ = & \text { igual a } \\ \mathrm{x} & \text { Vezes }\end{array}$




\section{LISTA DE FIGURAS}

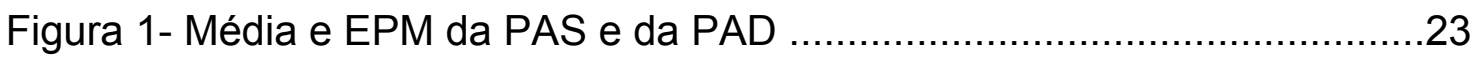

Figura 2 - Descenso sistólico (\%) antes e após paratireoidectomia ................25

Figura 3 - Descenso diastólico (\%) antes e após paratireoidectomia .............26

Figura 4 - Valores pré-operatórios e pós-operatórios de descenso

da PAS no pré-operatório, 6 e 12 meses ......................................27

Figura 5 - Valores pré-operatórios e pós-operatórios de descenso

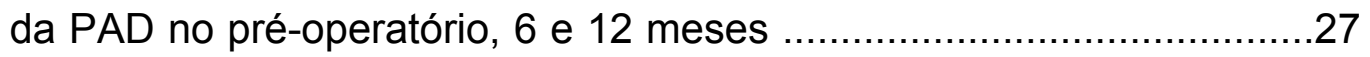

Figura 6 - Número de pacientes com descenso presente antes

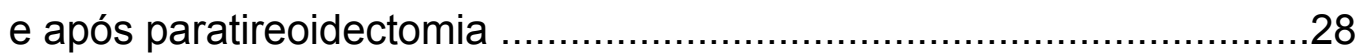




\section{LISTA DE TABELAS}

Tabela 1 - Parâmetros demográficos e laboratoriais das coortes inicial e final 18

Tabela 2 - Parâmetros pressóricos das coortes Inicial e Final.........................19

Tabela 3- Evolução do Cálcio total em miligramas por decilitro........................20

Tabela 4- Evolução do Cálcio ionizado em miligramas por decilitro..................20

Tabela 5- Evolução do Fósforo em miligramas por decilitro..............................21

Tabela 6- Evolução da Creatinina em miligramas por decilitro.........................21

Tabela 7- Evolução do PTH em picogramas por mililitro.................................22

Tabela 8 - Médias e erro padrão da média das medidas das pressões arteriais sistêmicas diurnas e noturnas 24

Tabela 9 - Uso de medicação anti-hipertensiva ao longo do período do estudo. .29 


\section{Resumo}

Massoni Neto LM. Avaliação da pressão arterial antes e após paratireoidectomia por hiperparatireoidismo primário [tese]. São Paulo: Faculdade de Medicina, Universidade de São Paulo; 2018.

INTRODUÇÃO: O hiperparatireoidismo primário (HPTP) é uma doença endócrina cuja prevalência aumentou muito nas últimas décadas. Descobriu-se grande contingente de portadores de formas leves e assintomáticos. Foi observado que esses pacientes apresentam alta morbidade e mortalidade por causas cardiovasculares. O estudo da pressão arterial revelou alta incidência de hipertensão arterial e descenso atenuado. A reversibilidade destes efeitos após a paratireoidectomia, entretanto é controversa. MÉTODO: Estudo prospectivo observacional com pacientes portadores de HPTP esporádico submetidos a paratireoidectomia para verificar as alterações dos parâmetros relativos à pressão arterial após a resolução do HPTP. Os pacientes realizaram monitorização ambulatorial de 24 horas da pressão arterial antes e após cirurgia curativa para HPTP, no $3^{\circ}$ pós-operatório, 3 meses, 6 meses e 12 meses. RESULTADOS: Em 7 casos (6 mulheres, idade média 65,7 anos), houve aumento do descenso da pressão arterial sistólica e diastólica. As médias (desvio padrão) da pressão arterial sistólica (PAS) e da pressão arterial diastólica (PAD) foram 124,0mmHg $(10,6)$ e $78,7 \mathrm{mmHg}(10,4)$. Não foi observada alteração significativa após a operação. As médias de PAS foram 129,4 mmHg (3 PO), 130,4 mmHg (3 meses) 125,4 mmHg(6 meses) e 131,1 $\mathrm{mmHg}$ (12 meses). As médias de PAD foram 73,7 mmHg (3 PO), 78,6 mmHg 
$75,4 \mathrm{mmHg}$ (6 meses) e 78,0 MMhg (12 meses). Por outro lado, o descenso noturno sistólico e diastólico da pressão arterial apresentaram melhora significativa aos 6 meses e sustentada aos 12 meses. As médias do descenso da PAS foram de $4,3 \%$ no pré-operatório; $1,2 \%$ no $3^{\circ}$ pós-operatório; $10,7 \%$ após 6 meses $(p=0,002)$ e de 10,5\% 12 meses depois da operação $(p=0,008)$. As médias do descenso da PAD foram de $7,1 \%$ no pré-operatório, $4,0 \%$ no $3^{\circ}$ pós-operatório, $13,3 \%$; aos 6 meses $(p=0,02)$ e de $14,7 \%$ depois de 12 meses da paratireoidectomia $(p=0,03)$. CONCLUSÃO: A paratireoidectomia melhora o descenso noturno da pressão arterial em pacientes com HPTP esporádico.

Descritores: hiperparatireoidismo primário; pressão sanguínea; monitorização ambulatorial da pressão arterial; paratireoidectomia; hipertensão; hipercalcemia 


\section{Abstract}

Massoni Neto LM. Arterial blood pressure monitoring before and after parathyroidectomy for primary hyperaparathyroidism [thesis]. São Paulo: "Faculdade de Medicina, Universidade de São Paulo", 2018

INTRODUCTION: Primary hyperparathyroidism is an endocrine disease and its prevalence has increased dramatically in the last decades. There is a great number of individuals with mild or asymptomatic forms of the disease. There is evidence of cardiovascular complications and mortality in these patients. The study of blood pressure showed high prevalence of hypertension and decreased dipping. The reversibility of these effects on blood pressure after curative parathyroidectomy is debatable. METHODS: Prospective study to evaluate the changes in blood pressure measurements of patients undergoing curative parathyroidectomy for sporadic PHPT with 24-hour ambulatory blood pressure monitoring before (PRE) and after curative surgery, during hospital stay (PO3), three months (3 mo), six months (6 mo) and at 12 months (12 mo). RESULTS: In 7 cases (6 female, mean age 65.7 years), there was an improvement of the nocturnal dipping of the systolic and diastolic arterial pressure. Mean (standard deviation) preoperative Systolic Blood Pressure (SBP) and Diastolic Blood Pressure (DBP) were $124.0 \mathrm{mmHg}(10.6)$ and 78.7 $\mathrm{mmHg}$ (10.4). No significant change in blood pressure was observed after the operation. Mean SBP values were $129.4 \mathrm{mmHg}$ (PO 3), $130.4 \mathrm{mmHg}$ (3 mo), $125.4 \mathrm{mmHg}(6 \mathrm{mo})$ and $131.1 \mathrm{mmHg}$ (12 mo). Mean DBP measures were $73.7 \mathrm{mmHg}$ (PO 3), $78.6 \mathrm{mmHg}$ (3 mo), $75.4 \mathrm{mmHg}$ (6 mo) and $78.0 \mathrm{mmHg}$ (12 
mo). Conversely, nocturnal systolic and diastolic dipping presented a small nonsignificant decrease immediately after the operation, but a statistically significant and sustained improvement at 6 months and 12 months. Mean systolic nocturnal dipping was $4.3 \%$ (PRE), $1.2 \%$ (PO 3); $10.7 \%$ (6 mo) $(p=0,002)$ and $10.5 \%(12 \mathrm{mo})(p=0.008)$. Mean diastolic nocturnal dipping was 7.1\% (PRE), 4.0\% (PO 3), 13.3\% (6 mo) $(p=0,01)$ and $14.7 \%(12 \mathrm{mo})(p=0.03)$. Conclusions: In sporadic PHPT, parathyroidectomy improves nocturnal dipping of blood pressure.

Descriptors: hyperparathyroidism, primary; blood pressure; blood pressure monitoring, ambulatory; parathyroidectomy; hypertension; hypercalcemia 


\section{INTRODUÇÃO}

O hiperparatireoidismo primário (HPTP) é uma doença endócrina caracterizada pela produção excessiva de paratormônio (PTH) pelas glândulas paratireoides, na ausência de estímulo metabólico para tal.

Quando presentes, os sintomas mais comuns são renais e ósseos, a saber, litíase urinária, nefrocalcinose, e perda da densidade mineral óssea. 0 diagnóstico de HPTP é feito quando há hipercalcemia PTH dependente, ou níveis de PTH inadequadamente elevados para os níveis de cálcio.

Estima-se que o HPTP afete cerca de $1 \%$ da população na Europa, e 0,86\% nos Estados Unidos da América ${ }^{2,3}$. Acomete principalmente mulheres após a sexta década de vida numa taxa que varia de 2 a 4 mulheres para cada homem, mas a incidência abaixo de 45 anos de idade é semelhante entre os sexos ${ }^{4}$. A prevalência de HPTP apresentou grande incremento a partir da década de 70 nos países desenvolvidos, com a adoção de detectores multicanal. Após esse período inicial de grande aumento no diagnóstico, houve um declínio no diagnóstico de casos novos dos anos 70 até os anos 90 , em decorrência do abandono da dosagem rotineira da calcemia ${ }^{5}$. A partir dos anos 2000, entretanto, a maior preocupação com a saúde óssea e a incorporação da densitometria óssea à prática clínica, além do maior conhecimento sobre o HPTP parecem ser responsáveis por manter alta a frequência de detecção da doença ${ }^{6}$.

A prevalência está associada com a apresentação clínica, isto é, em países onde a há alta incidência, a doença tende a ser predominantemente 
leve. Assim, em países desenvolvidos habitualmente existe alta prevalência de HPTP e apresentação predominantemente assintomática, enquanto em países cuja população tem menos acesso aos serviços de saúde ainda predomina a forma sintomática ${ }^{7}$.

No Brasil, também se observa o aumento progressivo no contingente de pacientes assintomáticos, em casuísticas de hospitais-escola. Enquanto na cidade de São Paulo notou-se aumento da proporção de casos assintomáticos de $11,2 \%$ na década de 80 para $47,7 \%$ nos anos $2000^{8}$, em Pernambuco foi relatada a existência de $81 \%$ de pacientes assintomáticos nos anos $2000^{9}$,

Uma forma ainda pouco conhecida é o denominada HPTP normocalcêmico, descrito inicialmente por Silverberg e Bilezikian em $2003^{10}$. O diagnóstico nesses casos geralmente é realizado no contexto de avaliação em serviços de metabolismo ósseo, e tem sido considerado uma fase inicial do HPTP, mas nem sempre é assintomático. Essa descrição levou a uma revisão do conceito clássico, onde julgava-se que os sintomas só ocorreriam em pacientes com hipercalcemia ${ }^{10}$. O manejo do HPTP normocalcêmico recomendado atualmente é o mesmo dispensado aos portadores de HPTP assintomático $^{11}$.

O tratamento cirúrgico é a única forma de cura definitiva do HPTP. Entretanto, em virtude da grande quantidade de pacientes assintomáticos, sem lesão dos órgãos alvo clássicos e, a partir da observação que muitos desses casos não apresentam progressão da doença durante observação de longo prazo ${ }^{12,13}$, propôs-se a conduta expectante para alguns indivíduos, observadas algumas premissas. Assim, em pacientes com HPTP assintomático, pode-se propor acompanhamento, desde que respeitadas 
algumas condições. Nos casos assintomáticos, a paratireoidectomia é fortemente recomendada para pacientes jovens (idade inferior a 50 anos), para aqueles em que haverá dificuldade para o seguimento proposto ou quando é observada lesão em órgãos-alvo, a saber: Depuração de creatinina menor que $60 \mathrm{~mL} / \mathrm{min}$, nefrocalcinose, nefrolitíase, osteoporose densitométrica (densidade mineral óssea $\leq 2,5$ desvios padrão em relação ao índice T em coluna lombar, bacia, colo femoral ou rádio distal, para mulheres após a menopausa ou homens acima dos 50 anos de idade) ou, presença de fratura de vértebra. Além disso, a operação é recomendável também quando o quadro laboratorial aponta Cálcio $1 \mathrm{mg} / \mathrm{dL}$ acima do limite superior do método, calciúria de 24 horas maior que $400 \mathrm{mg} / 24 \mathrm{~h}$ ou surgimento de sintomas durante o acompanhamento ${ }^{14-16}$.

Além das manifestações ósseas e renais do HPTP, a doença pode se manifestar em outros órgãos e sistemas. Pode haver fraqueza, fatigabilidade, ansiedade e prejuízo cognitivo ${ }^{1,17}$. Sintomas gastrintestinais como gastrite, obstipação, e até mesmo pancreatite são menos comuns e geralmente atribuídos à hipercalcemia ${ }^{18}$.

Em relação ao sistema cardiovascular, as possíveis manifestações clínicas do HPTP foram suspeitadas há bastante tempo. Em 1958, Hellström et al. mencionaram a possível associação entre HPTP e hipertensão arterial sistêmica com sugestão de que hipertensão arterial deveria ser uma das indicações de paratireoidectomia ${ }^{19}$. Em 1972, Rosenthal e Roy relataram alta incidência de hipertensão arterial sistêmica (32\%) entre os 40 pacientes diagnosticados com HPTP entre 1960 e 197120. Em 1973, Chowdhury et al. relataram a associação entre HPTP e hipertensão em uma série de 15 casos, 
atribuindo o aumento da pressão arterial à lesão renal desses pacientes, sendo que apenas um dos pacientes teve melhora após a paratireoidectomia ${ }^{21}$.

No entanto, em 1983, Jones et al. descreveram que na observação de 62 pacientes durante uma média de 2,9 anos após a paratireoidectomia houve alta incidência de doença renal crônica e hipertensão arterial, com piora após a operação. Nesse estudo, houve o desenvolvimento de hipertensão em $45 \%$ dos pacientes previamente normotensos e piora moderada da função renal em 9\% dos pacientes, com sugestão que a hipertensão arterial sistêmica observada em portadores de HPTP estaria mais relacionada às complicações renais determinada pela doença endócrina e não por ação direta ${ }^{22}$.

Apesar de não haver uma relação direta definida entre a hipertensão arterial sistêmica e HPTP, manteve-se a preocupação com as doenças cardiovasculares nessa população. Em estudo retrospectivo publicado em 1985, Ronni-Sivula et al. mostraram que em 334 pacientes operados entre 1956 e 1979 por HPTP houve alta morbimortalidade por causas cardiovasculares, quando comparado a pacientes operados no mesmo período por varizes, hemorroidas e apendicite. Os pacientes com HPTP morreram mais e mais jovens, apesar de sua operação ter sido realizada com a idade mais alta do que a do grupo controle. Porém, eles já apresentavam níveis mais elevados de creatinina à época da cirurgia. No momento da avaliação, já haviam falecido $55 \%$ dos pacientes com história de crise hipercalcêmica e $24 \%$ dos pacientes com osteitis fibrosa cystica (18 faleceram por infarto do miocárdio, 4 por acidente vascular cerebral, 4 por uremia e um por doença vascular periférica) Houve apenas 8 mortes no grupo controle no mesmo período, sugerindo algum efeito cardiovascular deletério atribuível ao HPTP ${ }^{23}$. 
Como a hipercalcemia é uma condição associada ao HPTP, a relação da mortalidade cardiovascular e a hipercalcemia foi salientada por Ljundhall et al., em 1991 Nesse ano, eles publicaram os resultados de um estudo prospectivo observacional, iniciado em 1969 e com 14 de anos de acompanhamento de 176 indivíduos hipercalcêmicos identificado em rastreamento de 24.171 indivíduos, em um distrito na Suécia. Observaram maior mortalidade do que o esperado nos indivíduos hipercalcêmicos (33, ante 23 esperadas), sendo que doenças cardiovasculares foram a maior causa das mortes. Desses pacientes, 39 foram operados por HPTP, e o demais, considerados casos leves, seguiram em observação ${ }^{24}$.

Em função de na atualidade o diagnóstico de HPTP ser determinado principalmente em indivíduos assintomáticos, nos quais a doença parece não progredir nos órgãos classicamente relacionados, o esclarecimento se de fato existirem complicações cardiovasculares independentes das lesões ósseas e renais foi salientado Silverberg, em 2002, em publicação referente a um dos primeiros encontros de especialista sobre o manejo de pacientes com o denominado HPTP assintomático. Essa autora destacou a necessidade de se estudar os órgãos alvo não clássicos do HPTP, em especial as manifestações neuropsiquiátricas e cardiovasculares. Isso porque não se sabe qual a extensão do acometimento dos diversos órgãos e sistemas afetados na apresentação contemporânea do HPTP, e, principalmente à luz da recomendação de acompanhamento para muitos destes pacientes ${ }^{25}$.

Alguns dados posteriores confirmaram a relevância de estudar doenças cardiovasculares em portadores de HPTP. Em 2003, Vestergaard et al. estudaram pacientes operados por HPTP de 1979 a 1997 e pacientes 
controles, com achado de risco aumentado (risco relativo [RR] 2,5) de infarto do miocárdio nos 10 anos anteriores à operação e no primeiro ano após o tratamento, com normalização do risco após $1 \mathrm{ano}^{26}$.

Em relação à pressão arterial sistêmica, Vaidya et al. publicaram em 2015 estudo utilizando uma grande coorte, com 75.000 enfermeiras, por meio de questionários e avaliação de prontuários médicos. Identificou um RR para HPTP entre mulheres hipertensas comparado com as não hipertensas de 1,80 . O RR foi ainda maior entre hipertensas em uso de furosemida $(1,88)$. O autor, propôs que o tratamento da hipertensão, por modular o manejo urinário de cálcio e sódio possa influenciar no desenvolvimento de HPTP. Em que pesem as limitações do uso de questionários, o grande número de participantes e a alta aderência garantem consistência, e a hipótese de certa maneira inverte a causalidade proposta na maior parte da literatura ${ }^{27}$.

No ano seguinte, Kalla et al. mostrou uma associação entre HPTP e hipertensão em um estudo populacional, em que avaliou os registros de uma grande base de dados em busca de associações entre HPTP e fatores de risco cardiovasculares. Baseado em quase oito milhões de prontuários, observou aumento na incidência de muitos fatores de risco cardiovasculares, como obesidade, hipertensão, diabetes mellitus, dislipidemia e doença renal crônica. Após análise multivariada, apenas a presença de hipertensão foi predita de forma independente por HPTP ${ }^{28}$.

Estudos com grandes populações permitiram demonstrar a associação de HPTP com hipertensão com consistência ${ }^{27,28}$. Entretanto, não permitem avaliar detalhes do comportamento da pressão arterial nesses pacientes. 
A monitorização ambulatorial da pressão arterial (MAPA) é o método que permite o registro indireto e intermitente da pressão arterial sistêmica durante 24 horas, ou mais, enquanto o paciente realiza suas atividades habituais na vigília e durante o sono. É uma ferramenta melhor do que a medida isolada da pressão, pois elimina o efeito do jaleco branco e permite a avaliação da variação circadiana da pressão ${ }^{29}$. A MAPA permite predizer com melhor acurácia o risco de eventos cardiovasculares, tais como infarto do miocárdio e acidente vascular cerebral, em relação à medida casual de consultório da pressão arterial sistêmica ${ }^{30}$. Um importante parâmetro avaliado pela MAPA é o descenso noturno na pressão. Trata-se da redução fisiológica da pressão arterial sistêmica durante o sono, de 10 a 20\% A ausência de descenso (descenso atenuado) é preditor de aumento do risco cardiovascular, associado a aumento de até $50 \%$ no risco de eventos cardiovasculares ${ }^{31,32}$.

Em 2002, Nuzzo et al. estudaram a espessura da camada íntima e da camada média da artéria carótida, da pressão arterial sistêmica por meio da MAPA e da massa ventricular esquerda, em 20 pacientes com HPTP e em 20 controles. Eles detectaram aumento da espessura das camadas na artéria carótida e não notaram qualquer diferença no comportamento da pressão arterial sistêmica entre os dois grupos ${ }^{33}$. Letizia et al. estudaram portadores de HPTP com MAPA, em 2005. Identificaram alta incidência de hipertensão arterial nesta população (47\%) e incidência aumentada de descenso atenuado entre os hipertensos portadores de HPTP em relação aos portadores de hipertensão essencial ${ }^{34}$. Não houve uma avaliação da pressão arterial sistêmica após o tratamento do HPTP nesses estudos, o que seria de grande relevância, para verificar se essas alterações seriam reversíveis. 
Em 2009 algumas pesquisas sobre o efeito da paratireoidectomia foram divulgadas. Na primeira, Heyliger et al. mostraram a redução dos níveis pressóricos sistólico e diastólico em mais de $10 \mathrm{mmHg}$ em pacientes hipertensos submetidos a paratireoidectomia, em estudo retrospectivo em que a pressão arterial sistêmica foi aferida antes e 6 meses após paratireoidectomia $^{35}$. Como grande limitação da validade dessa observação, ressalte-se que a pressão foi aferida uma única vez, durante a consulta regular do paciente, o que pode enfraquecer a qualidade dos dados obtidos para conclusões importantes. Em outra pesquisa publicada no mesmo ano, Bollerslev et al. mostraram os resultados de um ensaio clínico randomizado para operação ou observação de HPTP assintomático Não observaram melhora nos marcadores de doença cardiovascular e houve melhora dos níveis de pressão arterial média e da pressão diastólica, mas nos dois grupos ${ }^{36}$. Novamente, a metodologia de avaliação da pressão arterial sistêmica não foi claramente descrita e parece ter sido obtida por medidas isoladas.

Durante o desenvolvimento da presente pesquisa, aprovada em junho de 2010, outros dados sobre a avaliação da pressão arterial sistêmica em pacientes com HPTP foram publicados. Nesse mesmo ano, Feldstein et al. estudaram 46 pacientes operados por HPTP e acompanhados por uma média de 3,5 anos, com alta prevalência de hipertensão (54,35\%). Após a operação, observaram aumento da pressão em 5 indivíduos previamente normotensos. Os autores realizaram MAPA em 16 destes pacientes, apenas no pósoperatório, tendo observado relação entre o cálcio total e pressão sistólica noturna, e descenso atenuado da pressão arterial. Identificaram também depuração de creatinina mais elevada em pacientes com descenso presente, 
além de alta prevalência de descenso atenuado ${ }^{37}$ Apesar de terem aplicado a MAPA, a metodologia não permitiu avaliação adequada da influência da operação numa eventual melhora.

Essa dúvida seria melhor esclarecida ainda no ano de 2010. Rydberg et al. encontraram aumento discreto da pressão arterial sistêmica entre os hipertensos, e redução discreta entre os não hipertensos com medidas de 24 horas da pressão arterial, analisadas pela MAPA, em 49 portadores de HPTP antes e 6 meses após paratireoidectomia ${ }^{38}$. Apesar da excelente metodologia, infelizmente ainda não ficou claro se essa melhora ocorreu rapidamente após a operação ou mais tardiamente.

Em 2011 adicionaram-se possíveis sugestões de melhora da pressão arterial sistêmica após a paratireoidectomia. Broulik et al. relataram a redução das medidas de pressão arterial sistólica e diastólica 6 meses após paratireoidectomia por HPTP em 1020 pacientes operados. Infelizmente, as medidas de pressão foram obtidas com medidas simples com esfigmomanômetro em consulta ambulatorial e persistiu a dúvida sobre qual o momento dessa melhora ${ }^{39}$.

Em 2012, Petramala et al. publicaram resultados da avaliação de 30 pacientes com HPTP, 30 hipertensos e 30 controles hígidos, por meio da MAPA, Ecocardiograma e Doppler arterial colorido antes e 1 ano após paratireoidectomia. Observaram no grupo operado redução da prevalência de síndrome metabólica, redução da pressão arterial e aumento do descenso noturno $^{40}$. Em 2013 houve publicação de outro estudo com MAPA antes e 1 ano após paratireoidectomia. Avaliando 150 pacientes, detectou-se redução da pressão sistólica 1 ano após paratireoidectomia, sem diferença entre grupos 
que receberam suplementação de vitamina $D$ ou não ${ }^{41}$. Analisando a mesma coorte de 150 pacientes, posteriormente foi publicado artigo em que foi pesquisada a correlação entre FGF23 e risco cardiovascular, tendo sido identificada associação entre os níveis de FGF23 no pré-operatório com a massa do adenoma de paratireoide e níveis de PTH, além de triglicérides, níveis de insulina e índice de massa corpórea. Foi observada também, após análise multivariada, correlação entre os níveis de PTH e a pressão sistólica noturna ${ }^{42}$. Ressalte-se a excelente metodologia empregada nesses estudos, mas ainda não há evidência se essa melhora ocorreria precocemente após a operação ou em longo prazo.

Em 2017, Concistrè et al. publicaram estudo com MAPA em que avaliaram 25 pacientes com HPTP, dos quais 15 foram reavaliados após 1,9 anos em média após paratireoidectomia. Apesar de não ter detectado mudanças na pressão arterial sistólica e diastólica globais, diurnas e noturnas 2 anos após a operação, observou melhora (redução) da variabilidade da pressão arterial, parâmetro até então não estudado em HPTP ${ }^{43}$. No mesmo ano, Storvall et al.publicaram um estudo com avaliação de 104 pacientes após paratireoidectomia, (3,3 anos em média) em que avaliaram por meio de questionários, a qualidade de vida relacionada à saúde e a medida de pressão arterial sistêmica auto-relatada. Observaram melhora da cognição, sono e redução da pressão. Constitui, apesar das limitações do método, uma das avaliações de mais longo prazo da pressão arterial sistêmica após paratireoidectomia, e mostra que a redução da pressão arterial persiste por longo prazo $0^{44}$.

Desse modo, verifica-se que a pesquisa da pressão arterial sistêmica 
em pacientes com HPTP tem grande importância clínica. Há evidências não totalmente consolidadas de possível melhora de parâmetros mensurados.

Fica evidente que ainda não há estudos publicados que tenham analisado as variações da pressão arterial sistêmica pela MAPA, comparando a condição pré-operatória com estados pós-operatórios precoces e tardios. 


\section{OBJETIVO}

Analisar o comportamento da pressão arterial sistêmica nos períodos pré-operatório, e pós-operatório de paratireoidectomia por hiperparatireoidismo primário esporádico, por meio de medidas ambulatoriais de 24 horas, e verificar qual é o comportamento da pressão arterial sistêmica nesses pacientes, se existem modificações nos parâmetros relativos a medidas da pressão arterial sistêmica no terceiro dia após a operação, 3 meses, 6 meses e 12 meses após paratireoidectomia. 


\section{MÉTODO}

\subsection{Desenho do estudo}

Estudo prospectivo observacional das medidas de pressão arterial sistêmica por MAPA, de fevereiro de 2011 até janeiro de 2013, com pacientes operados por HPTP esporádico pelo Grupo de Paratireoides e Doenças Congênitas do Serviço de Cirurgia de Cabeça e Pescoço da Divisão de Clínica Cirúrgica 1 das Unidades Médicas e de Apoio do Hospital das Clínicas da Faculdade de Medicina da Universidade de São Paulo (HCFMUSP), aprovado pelo comitê de ética em pesquisa da instituição com Certificado de Apresentação para Avaliação Ética (CAEE) número 0353.0.015.000-10 (anexo C),. Esses pacientes foram provenientes e acompanhados nos ambulatórios do próprio serviço bem como do Serviço de Endocrinologia do mesmo hospital.

\subsection{Critérios de Inclusão:}

Pacientes maiores de 18 anos, , com diagnóstico de HPTP e indicação de paratireoidectomia, com aceitação de realizar os exames previstos, função renal pré-operatória sem diagnóstico de doença renal crônica estágios 3b, 4 ou 5 e que, após esclarecimento, concordaram em participar do estudo e assinaram o termo de consentimento

\subsection{Critérios de Exclusão:}

Excluíram-se os pacientes com diagnóstico de HPTP familiar, os pacientes que apresentaram persistência do hiperparatireoidismo, definida como não resolução duradoura da hipercalcemia e com nível inadequado de 
PTH até 6 meses após a operação e também os pacientes que não realizaram a MAPA de forma adequada em mais de uma ocasião prevista no protocolo.

3.4 Exames laboratoriais:

Todos os pacientes realizaram os exames laboratoriais de acompanhamento já constantes da rotina de cuidado assistencial. em tempos determinados, a saber, pré-operatório, na semana anterior à operação, terceiro dia pós-operatório, ainda internados, 3 meses, 6 meses, e 1 ano após a cirurgia, conforme rotina assistencial do serviço. Os métodos laboratoriais utilizados para medida de cálcio total (CaT) e cálcio ionizado (Cai) foram, respectivamente, calorimétrico automatizado e eletrodo íon seletivo, cujos valores de referência são, respectivamente, 8,6 a 10,2 $\mathrm{mg} / \mathrm{dL}$ e 4,6 a 5,3 $\mathrm{mg} / \mathrm{dL}$. O fósforo $(\mathrm{P})$ foi medido através do método enzimático colorimétrico automatizado com valores de referência entre 2,7 a $4,5 \mathrm{mg} / \mathrm{dL}$. A Creatinina (Cr) foi medida com o ensaio colorimétrico cinético baseado no método Jaffé, cujos valores de referência de $0,70-1,20 \mathrm{mg} / \mathrm{dL}$ para homens e 0,50-0,90 $\mathrm{mg} / \mathrm{dL}$ para mulheres. $\mathrm{O}$ PTH foi medido por método imunoquimioluminométrico, no equipamento IMMULITE 2000 com kits da fornecedora SIEMENS, cujos valores de referência eram 16 a $87 \mathrm{pg} / \mathrm{mL}$ e sensibilidade de $3 \mathrm{ng} / \mathrm{mL}$, e após 21/11/2012 houve mudança do método para ensaio de quimioluminescência com kits da fornecedora Corelab, cujos valores de referência eram 15 a $65 \mathrm{pg} / \mathrm{mL}$. Os exames foram processados pelas Divisão de Laboratório Central do HCFMUSP e disponibilizados no sistema de prontuário eletrônico da instituição. 
A participação no estudo não levou a mudanças no tratamento cirúrgico ou no manejo pós-operatório destes indivíduos (estudo observacional).

\subsection{Procedimento cirúrgico}

A operação foi realizada de acordo com a rotina da instituição. Resumidamente, a cirurgia foi direcionada para uma única glândula sempre que possível, de acordo com os dados clínicos e de imagem, sob anestesia geral e com dosagens intra-operatórias de PTH, utilizando o critério de Miami modificado, caracterizado pela queda de $50 \%$ ou mais na coleta após 10 minutos em relação ao maior valor obtido antes da retirada da paratireoide suspeita $^{45}$. A queda insatisfatória do PTH intra-operatório conduziu a exploração bilateral.

\subsection{MAPA}

As medidas foram realizadas utilizando o equipamento Spacelabs $90207^{46}$ Um manguito apropriado à circunferência do braço foi instalado em membro não dominante. Medidas de pressão arterial foram realizadas a cada 20 minutos durante a vigília e 30 minutos durante o sono. Os períodos de sono e vigília foram ajustados caso a caso segundo informações fornecidas pelos pacientes. Cada MAPA foi aceita mediante pelo menos 16 medidas em vigília e $8 \mathrm{em}$ sono $^{47}$. Foram avaliadas as médias de pressão arterial sistólica (PAS), pressão arterial diastólica (PAD) em 24 horas, em vigília e dormindo. 
Para efeito de análise, os pacientes foram definidos como hipertensos em caso PAS> $140 \mathrm{mmHg}$ ou PAD > 90mmHg à avaliação inicial, ou diagnóstico prévio de hipertensão arterial sistêmica. Pacientes em uso de medicação anti-hipertensiva com outra finalidade foram analisados juntamente com hipertensos em tratamento. O descenso noturno foi calculado de acordo com a seguinte fórmula (para PAS e PAD):

(Média da Pressão Arterial em vigília - Média da Pressão Arterial sono X 100 Média da Pressão Arterial em vigília

Classificou-se em descenso presente para descenso noturno maior que $9 \%$ e em descenso atenuado para descenso noturno menor ou igual a $9 \%{ }^{31}$. O protocolo incluiu o registro de MAPA na semana anterior à operação, no terceiro dia pós operatório, após 3, 6 e 12 meses.

\subsection{Estatística}

A análise estatística foi realizada com os softwares MINITAB $16 \AA$ e GraphPad Prism ®. Variáveis discretas são apresentadas como contagem e frequência. Elas foram comparadas utilizando o teste exato de Fisher. Todas as variáveis contínuas foram testadas para normalidade com o teste Anderson Darling e estão apresentadas como média $(\mathrm{m}) \pm$ erro padrão da média (EPM), se a distribuições testadas eram normais. Em caso de distribuição não paramétrica, estão apresentadas como mediana e intervalo interquartis (Q1Q3). O teste $\mathrm{t}$ não pareado ou pareado foram usados quando adequado, assim como Análise de Variância (ANOVA) e ANOVA com Medidas Repetidas. Distribuições não paramétricas foram comparadas com teste de Mann-Whitney ou Friedman e teste de Wilcoxon. 


\section{RESULTADOS}

A coorte inicial incluiu 22 pacientes com HPTP esporádico. Todos tiveram pelo menos uma indicação de cirurgia. Sete pacientes completaram a avaliação de 12 meses.

Dos 15 pacientes excluídos, um teve HPTP persistente apesar da queda adequada do PTH intra-operatório. Todas as demais exclusões (14) foram por abandono do protocolo. Uma paciente removeu o manguito do monitor na noite do primeiro exame, e assim teve dados iniciais incompletos, sem registros noturnos. Outros 3 pacientes abandonaram o protocolo após o primeiro exame, e mais 3 pacientes após o segundo MAPA (realizado no 3PO), outros 2 pacientes desistiram de participar após o terceiro exame, (realizado 1 semana após a operação). Aos 6 meses, mais 2 pacientes abandonaram o protocolo e aos 12 meses outros 3 pacientes não realizaram o exame.

As Tabelas 1 e 2 mostram as características de ambos os grupos no início do estudo. Os parâmetros demográficos bem como valores da média do CaT, Cai, P, PTH e Cr no pré-operatório, com os respectivos valores do EPM, encontram-se na Tabela 1. Os parâmetros pressóricos estão apresentados na Tabela 2. Não houve diferença estatisticamente significativa entre os grupos.

A evolução dos parâmetros bioquímicos está demonstrada nas Tabelas de 3 a 7. Além dos efeitos esperados de redução dos valores de cálcio e PTH, observamos pequeno aumento (não significativo) da creatinina logo após o procedimento, com posterior normalização. 
Tabela 1 - Parâmetros demográficos e laboratoriais das coortes Inicial e Final

\begin{tabular}{|c|c|c|c|}
\hline & COORTE INICIAL & COORTE FINAL & $\begin{array}{c}\text { Nível } \\
\text { descritivo }\end{array}$ \\
\hline $\mathrm{n}$ & 22 & 7 & - \\
\hline Idade (anos) & $61,2 \pm 10,2$ & $66,7 \pm 3,0$ & $p=0,260$ \\
\hline Gênero (Mulheres / Homens) & $20 / 2$ & $6 / 1$ & $p=1,00$ \\
\hline $\begin{array}{l}\text { Presença de Hipertensão / } \\
\text { Uso de Medicação }\end{array}$ & $11(50 \%)$ & $5(72 \%)$ & $p=1,00$ \\
\hline PTH Mediana (Q1-Q3) pg/mL & $170(116-262)$ & $121(108-226)$ & $p=0,42$ \\
\hline Ca T (mg/dL) & $10,8 \pm 1,1$ & $10,3 \pm 1,2$ & $p=0,379$ \\
\hline Ca i (mg/dL) & $6,0 \pm 0,18$ & $5,8 \pm 0,32$ & $p=0,89$ \\
\hline$P(\mathrm{mg} / \mathrm{dL})$ & $2,8 \pm 0,13$ & $3,0 \pm 0,24$ & $p=0,75$ \\
\hline $\mathrm{Cr}(\mathrm{mg} / \mathrm{dL})$ & $0,9 \pm 0,05$ & $1,0 \pm 0,06$ & $p=0,34$ \\
\hline
\end{tabular}


Tabela 2 - Parâmetros pressóricos das coortes Inicial e Final

\begin{tabular}{|c|c|c|c|}
\hline & COORTE INICIAL & COORTE FINAL & $\begin{array}{c}\text { Nível } \\
\text { descritivo }\end{array}$ \\
\hline $\mathrm{n}$ & 22 & 7 & - \\
\hline PAS (mmHg) & $128,7 \pm 11,5$ & $124,0 \pm 10,6$ & $p=0,342$ \\
\hline PAD (mmHg) & $78,7 \pm 10,4$ & $72,9 \pm 7,2$ & $p=0,113$ \\
\hline PAS diurna $(\mathrm{mmHg})$ & $131,4 \pm 12,2$ & $128,1 \pm 11,6$ & $p=0,325$ \\
\hline PAS noturna (mmHg) & $120,1 \pm 11,1$ & $120,4 \pm 11,2$ & $p=0,954$ \\
\hline PAD diurna $(\mathrm{mmHg})$ & $81,5 \pm 10,1$ & $74,7 \pm 7,5$ & $p=0,081$ \\
\hline PAD noturna $(\mathrm{mmHg})$ & $70,5 \pm 9,6$ & $69,3 \pm 7,8$ & $p=0,734$ \\
\hline Descenso sistólico(\%) & $7,7 \pm 6,93$ & $4,3 \pm 7,6$ & $p=0,319$ \\
\hline $\begin{array}{l}\text { Descenso } \\
\text { diastólico(\%) }\end{array}$ & $12,4 \pm 8,08$ & $7,1 \pm 7,7$ & $p=0,149$ \\
\hline $\begin{array}{l}\text { n Descenso sistólico } \\
\text { presente }\end{array}$ & $7 / 15$ & $2 / 5$ & $p=1,0$ \\
\hline $\begin{array}{l}\text { n Descenso diastólico } \\
\text { presente }\end{array}$ & $14 / 8$ & $2 / 5$ & $p=0,19$ \\
\hline
\end{tabular}

NOTA: PAS (pressão arterial sistólica), PAD (pressão arterial diastólica), mmHg (milímetros de mercúrio) 
Tabela 3: Evolução do Cálcio total em miligramas por decilitro

\begin{tabular}{cccccc}
\hline CASO & PRE & 3 PO & 3 MESES & 6 MESES & 12 MESES \\
A & 10,4 & 7,6 & 9,4 & 8,9 & 8,6 \\
B & 9,3 & 8,3 & 9,7 & 8,8 & 8,6 \\
C & 9,7 & 8,3 & 9,4 & 8,9 & 8,9 \\
D & 11,8 & 9,8 & 10 & 9,4 & 9,8 \\
E & 9,4 & 8,5 & 9,8 & 11,11 & 9,2 \\
F & 9,6 & 9 & 9,3 & 9,1 & 8,8 \\
G & 12,2 & 9 & & 10,4 & 9,9 \\
\hline Média & 10,34 & 8,64 & 9,6 & 9,52 & 9,11 \\
EPM & 0,45 & 0,26 & 0,11 & 0,34 & 0,21 \\
\hline
\end{tabular}

NOTA: PRE (pré-operatório), 3PO ( terceiro pós-operatório) EPM (erro padrão da média)

Tabela 4: Evolução do Cálcio ionizado em miligramas por decilitro

\begin{tabular}{|c|c|c|c|c|c|}
\hline CASO & PRÉ & $3 \mathrm{PO}$ & 3 MESES & 6 MESES & 12 MESES \\
\hline A & 5,20 & 4,20 & 5,00 & 3,66 & 4,74 \\
\hline B & 5,41 & 4,80 & 4,95 & 4,76 & 4,85 \\
\hline C & 5,40 & 4,40 & 4,58 & 5,16 & 4,95 \\
\hline D & 6,40 & 5,50 & 5,30 & 5,10 & 5,07 \\
\hline$E$ & 5,40 & 4,40 & 4,80 & 5,50 & 5,00 \\
\hline $\mathrm{F}$ & 5,20 & 4,50 & 4,70 & 4,52 & 4,67 \\
\hline G & 7,50 & 5,20 & & 4,80 & 4,83 \\
\hline Mediana & 5,4 & 4,5 & 4,875 & 4,8 & 4,85 \\
\hline Q1-Q3 & $5,2-6,4$ & $4,4-5,2$ & $4,67-5,08$ & $4,52-5,16$ & $4,74-5,0$ \\
\hline
\end{tabular}


Tabela 5: Evolução do fósforo em miligramas por decilitro

\begin{tabular}{cccccc}
\hline CASO & PRE & 3 PO & 3 MESES & 6 MESES & 12 MESES \\
A & 3,20 & 4,30 & 3,90 & 3,4 & 4,5 \\
B & 2,60 & 4,20 & 3,40 & 3,00 & 2,80 \\
C & 3,40 & 2,60 & 2,90 & 2,70 & 2,50 \\
D & 2,40 & 4,00 & 3,30 & 3,70 & 4,50 \\
E & 3,70 & 4,40 & 3,50 & 5,40 & 3,20 \\
F & 3,50 & 4,80 & 4,20 & 4,30 & 4,20 \\
G & 2,00 & 3,40 & & 3,00 & 2,80 \\
\hline Média & 2,97 & 3,96 & 3,53 & 3,64 & 3,5 \\
EPM & 0,24 & 0,28 & 0,19 & 0,36 & 0,33 \\
\hline
\end{tabular}

NOTA: PRE (pré-operatório), 3PO ( terceiro pós-operatório) EPM (erro padrão da média)

Tabela 6: Evolução da Creatinina em miligramas por decilitro

\begin{tabular}{cccccc}
\hline CASO & PRE & $3 P O$ & 3 MESES & 6 MESES & 12 MESES \\
A & 1,05 & 0,91 & 1,24 & 1,08 & 0,99 \\
B & 1,20 & 1,56 & 1,13 & 1,10 & 1,09 \\
C & 0,94 & 1,17 & 0,95 & 0,75 & 0,87 \\
D & 0,82 & 1,39 & 0,71 & 0,82 & 0,87 \\
E & 0,88 & 0,93 & 0,92 & 1,21 & 0,83 \\
F & 1,18 & 1,39 & 1,11 & 1,24 & 1,08 \\
G & 0,92 & 1,04 & & 0,89 & 0,99 \\
\hline Média & 1,00 & 1,20 & 1,01 & 1,01 & 0,96 \\
EPM & 0,06 & 0,10 & 0,08 & 0,07 & 0,04 \\
\hline
\end{tabular}

NOTA: PRE (pré-operatório), 3PO ( terceiro pós-operatório) EPM (erro padrão da média), 
Tabela 7: Evolução do PTH em picogramas por mililitro

\begin{tabular}{|c|c|c|c|c|c|}
\hline CASO & PRE & $3 \mathrm{PO}$ & 3 MESES & 6 MESES & 12 MESES \\
\hline$A$ & 108 & 0 & 35 & 55 & 54 \\
\hline$B$ & 145 & 49 & 75 & 82 & 113 \\
\hline $\mathrm{C}$ & 100 & 12 & 44 & 42 & 92 \\
\hline$D$ & 226 & & 31 & 50 & 36 \\
\hline$E$ & 121 & 10 & 56 & 6 & 54 \\
\hline$F$ & 110 & 0 & & 45 & 51 \\
\hline$G$ & 314 & & & 84 & 52 \\
\hline Mediana & 121 & 10 & 44 & 50 & 54 \\
\hline Q1-Q3 & $108-226$ & $0-30,5$ & $33-65,5$ & $42-82$ & $51-92$ \\
\hline
\end{tabular}

Em relação aos sete casos com avaliação completa da MAPA, não houve diferenças significativas na PAS e PAD ao longo do estudo (Figura 1, Tabela 4). As medidas descritivas das observações diurnas e noturnas estão explicitadas na Tabela 8. Não houve alteração estatisticamente significativa. 


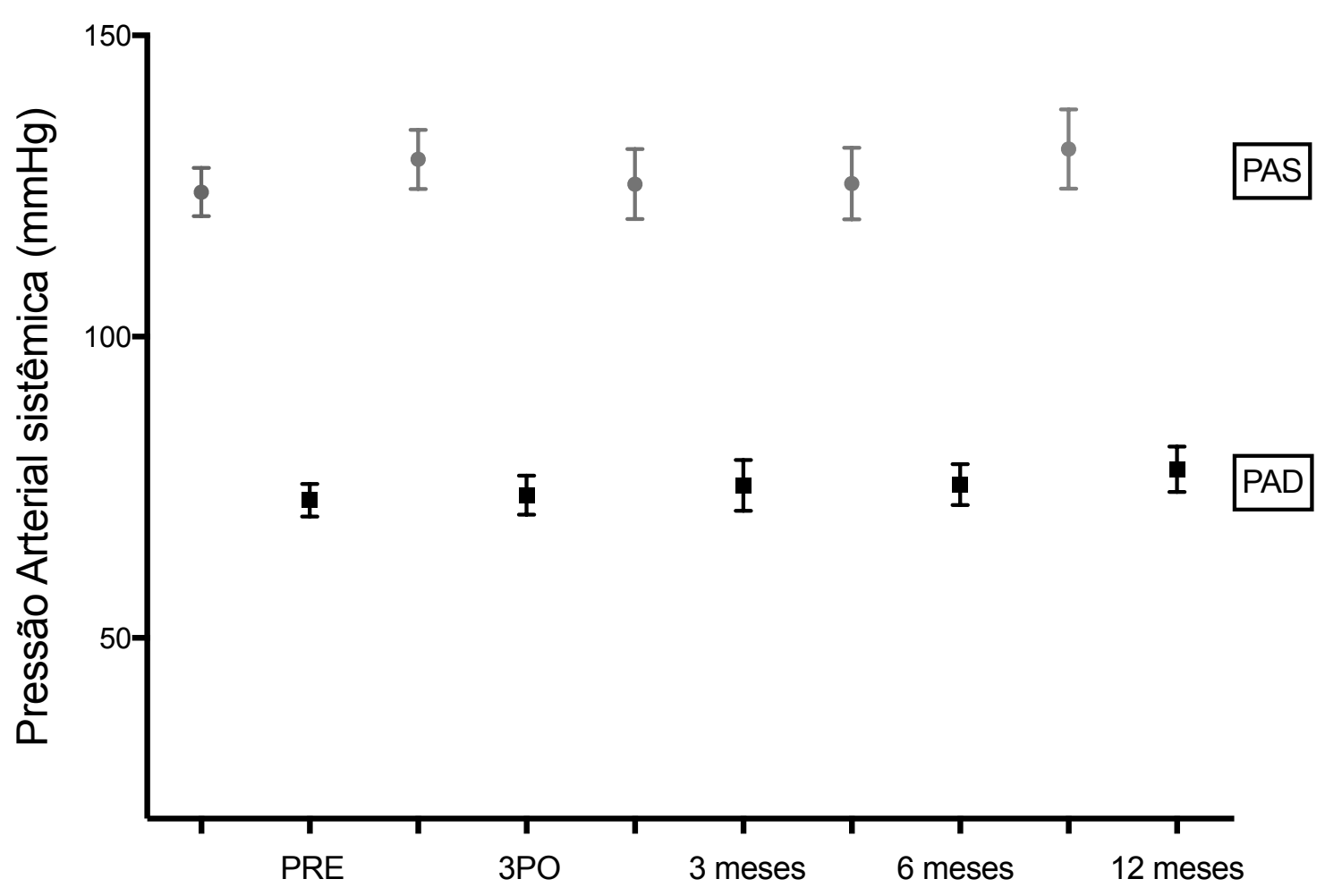

Figura 1- Média e EPM da PAS e da PAD 
Tabela 8 - Médias e erro padrão da média das medidas das pressões arteriais sistêmicas diurnas e noturnas

$\begin{array}{lllll}\text { PRE } & 3 \mathrm{PO} & 3 \mathrm{MESES} & 6 \text { MESES } & 12 \text { MESES }\end{array}$

PAS diurna $(\mathrm{mmHg})$

$$
126,1 \pm 4,4 \quad 131,5 \pm 4,8 \quad 131,5 \pm 7,5 \quad 129,3 \pm 7,5 \quad 134,9 \pm 6,6
$$

PAS

noturna $\quad 120,4 \pm 4,2 \quad 128,8 \pm 4,6 \quad 120,0 \pm 8,9 \quad 115,1 \pm 5,2 \quad 120,7 \pm 7,6$ $(\mathrm{mmHg})$

PAD diurna $(\mathrm{mmHg})$

$$
74,7 \pm 2,8 \quad 77,3 \pm 4 \quad 79,0 \pm 4,4 \quad 78,3 \pm 3,6 \quad 81,4 \pm 3,6
$$

PAD

noturna $\quad 69,3 \pm 2,8 \quad 73,6 \pm 3,6 \quad 68,7 \pm 6,2 \quad 67,7 \pm 3,8 \quad 69,7 \pm 5,3$ $(\mathrm{mmHg})$

NOTA: PRE (pré-operatório), 3PO ( terceiro pós-operatório),PAS (pressão arterial sistólica), PAD (pressão arterial diastólica), $\mathrm{mmHg}$ (milímetros de mercúrio)

Tanto o descenso sistólico quanto o diastólico apresentaram uma pequena redução não significativa na primeira medida após a cirurgia, mas melhoraram. Posteriormente, ocorreu aumento sustentado do descenso (figuras 3 e 4). Aos 6 meses e 12 meses houve diferença significativa tanto do descenso da PAS quanto da PAD em relação ao pré-operatório (teste $t$ pareado, PAS $p=0.0017$ aos 6 meses e $p=0,0080$ aos 12 meses e PAD $p=0,00131$ aos 6 meses e $p=0,00302$ aos 12 meses), A média do descenso da PAS aumentou de $4,3 \%$ no pré-operatório para $10,7 \%$ aos 6 meses e 10,5\% 
aos 12 meses. Para o descenso da PAD, o aumento foi ligeiramente maior, de $7,1 \%$ no pré-operatório para $13,4 \%$ aos 6 meses e $14,7 \%$ aos 12 meses .

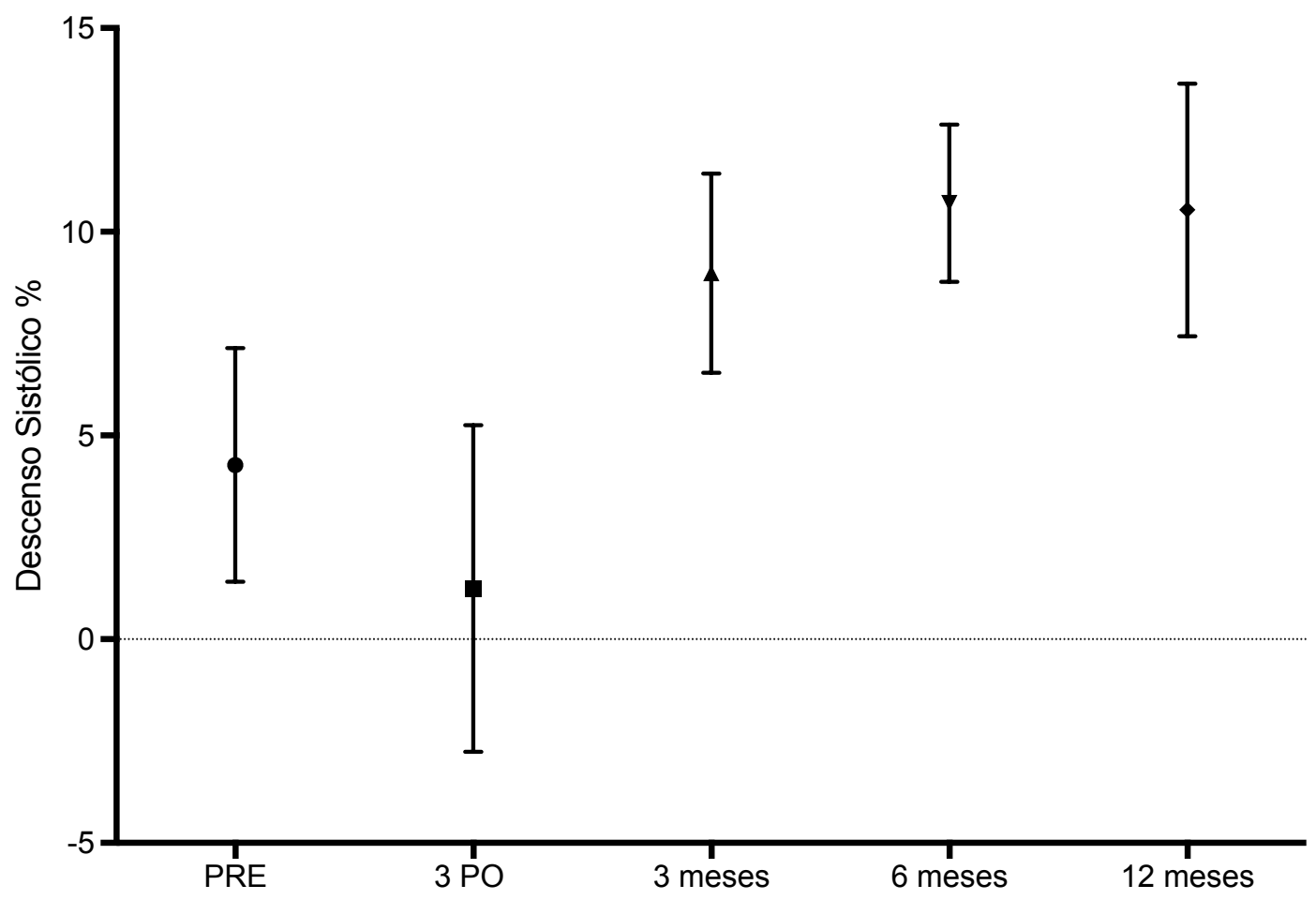

Figura 2 - Descenso sistólico (\%) antes e após paratireoidectomia 


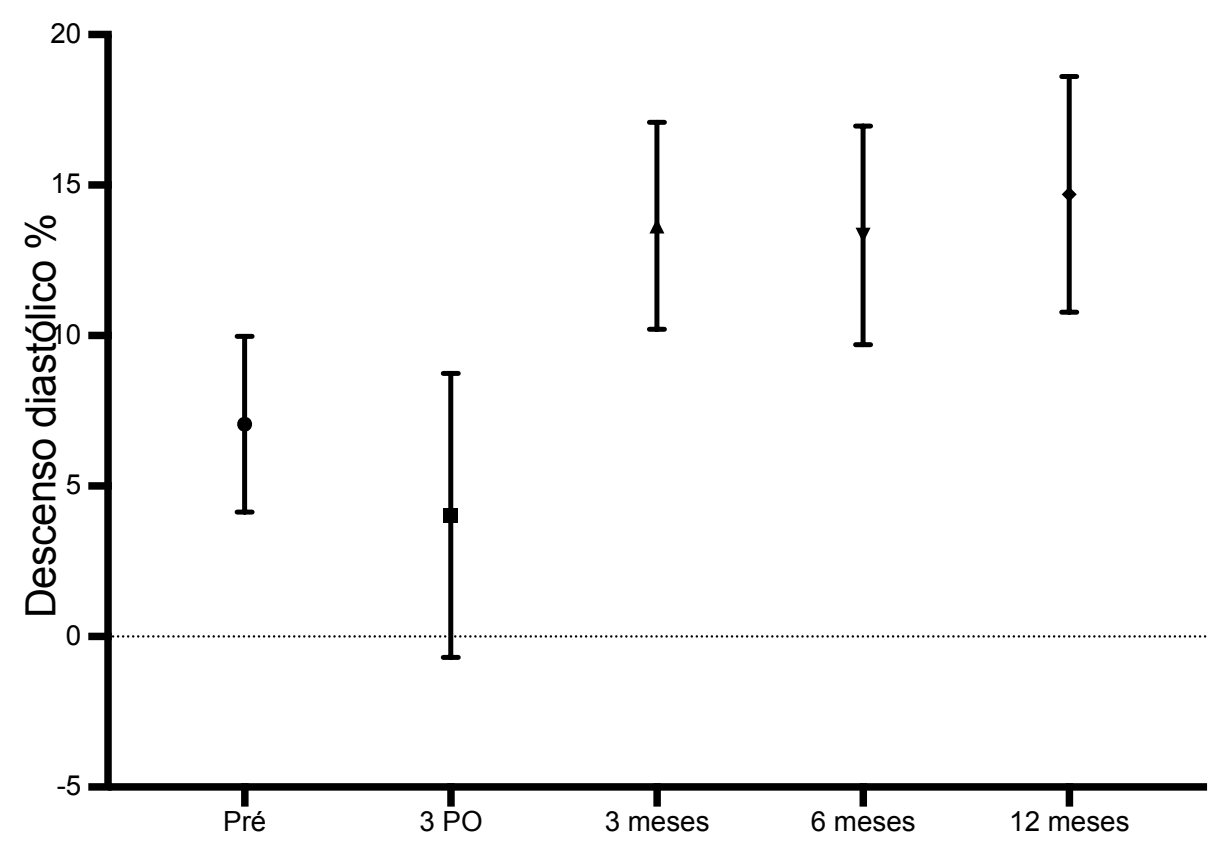

Figura 3 - Descenso diastólico (\%) antes e após paratireoidectomia

A Figuras 4 e 5 represetam os valores individuais calculados dos descensos da PAS e PAD no pré-operatorio, aos seis meses e aos 12 meses.

Decorridos 12 meses após a operação curativa, houve aumento não significativo da proporção de pacientes com descenso presente, de 28,6\% para $85,7 \%$ para descenso da PAS $(p=0,59)$ e de $28,6 \%$ para $85,77 \%$ para descenso da PAD $(p=0,10)$ (Figura 6). 


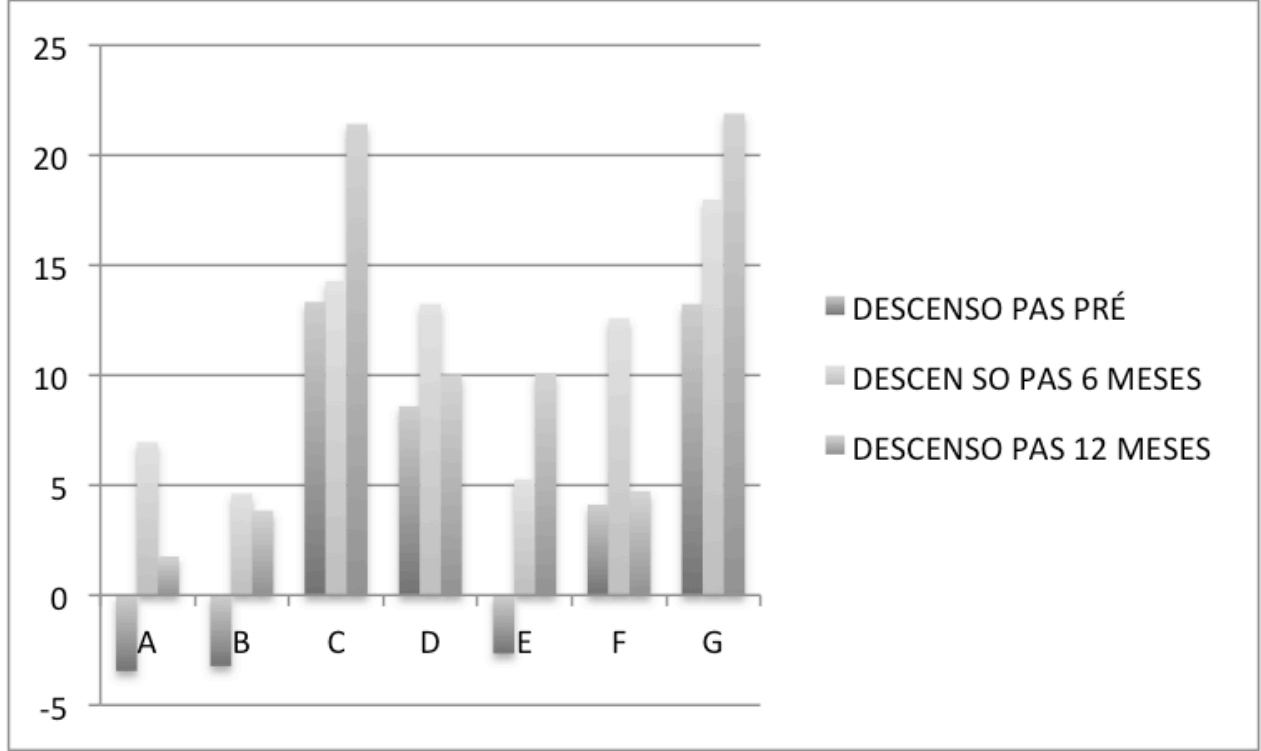

Figura 4 - Valores do descenso da PAS no pré-operatório, 6 e 12 meses

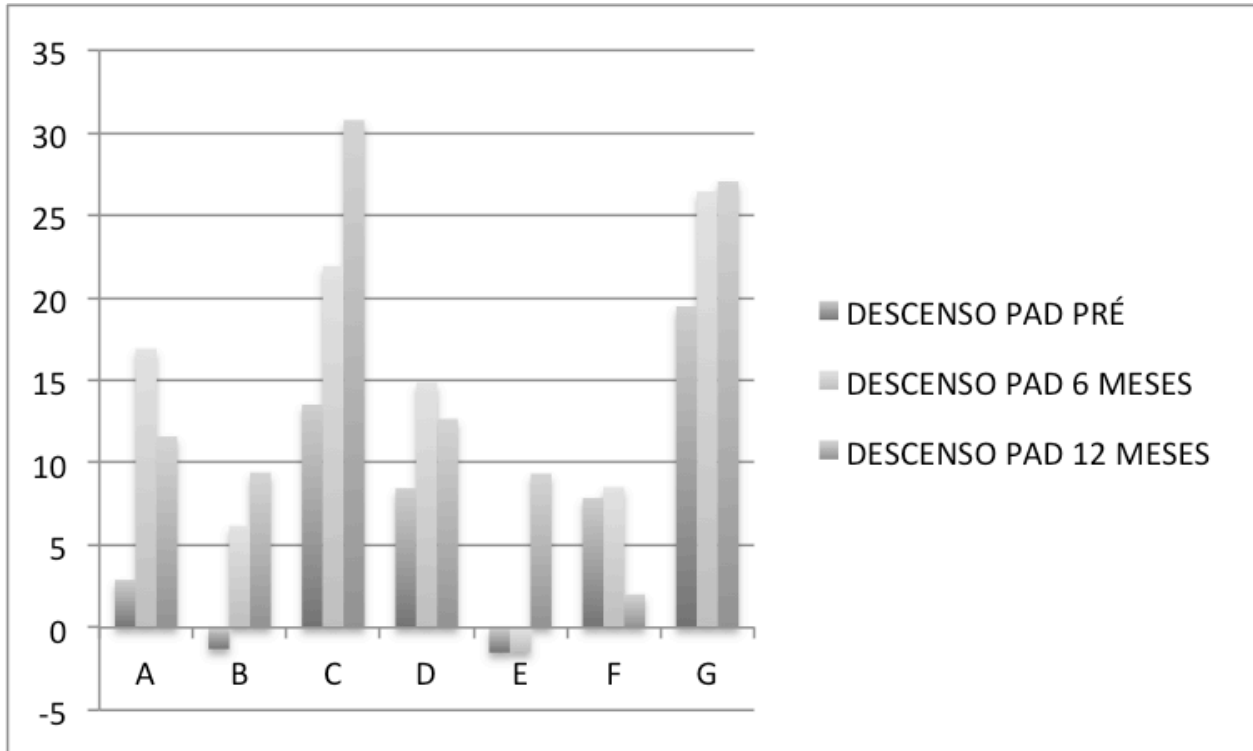

Figura 5 - Valores do descenso da PAD no pré-operatório, 6 e 12 meses 

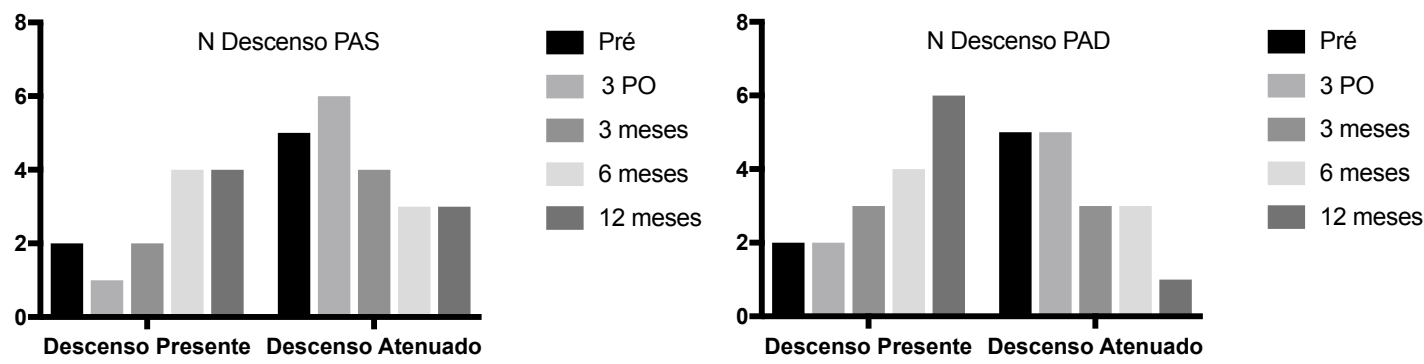

Figura 6 - Número de pacientes com descenso presente antes e após paratireoidectomia

A fim de avaliar se os efeitos observados poderiam ter sido causados por alteração de tratamento anti-hipertensivo dos pacientes, estudou-se a utilização de anti-hipertensivos. Dois casos não faziam uso de medicação antihiperteniva e assim permaneceram durante o acompanhamento (CASOS A e B) Um caso não era hipertenso, fazia uso de furosemida por edema e manteve a medicação durante todo o acompanhamento (CASO C). No caso D, o médico responsável diminuiu o número de medicações anti-hipertensivas em uso (de 3 para 2 drogas), sem incremento da dose das demais. Dois casos trocaram a monoterapia em uso (CASOS E e F), e o caso G apresentou acréscimo de uma droga (de 1 para 2 drogas). A Tabela 9 mostra o uso de medicações anti-hipertensiviva e as figura 4 e 5 mostram o comportamento dos descensos sistólico e diastólico de todos os casos a fim de ilustrar graficamente o descenso em cada caso. Não realizou-se inferência estatística em subgrupos devido ao pequeno número de casos. 
Tabela 9 - Uso de medicação anti-hipertensiva ao longo do estudo

\begin{tabular}{|c|c|c|c|c|}
\hline CASO & MED PRE & MED 3M & MED 6M & MED 12M \\
\hline A & 0 & 0 & 0 & 0 \\
\hline B & 0 & 0 & 0 & 0 \\
\hline C & diurérico tiazídico & diurérico tiazídico & diurérico tiazídico & diurérico tiazídico \\
\hline D & $\begin{array}{c}\text { diurérico tiazídico + } \\
\text { betabloqueado + } \\
\text { antagonista dos } \\
\text { receptores da } \\
\text { angiotensina }\end{array}$ & $\begin{array}{c}\text { diurérico tiazídico + } \\
\text { antagonista dos } \\
\text { receptores da } \\
\text { angiotensina }\end{array}$ & $\begin{array}{c}\text { diurérico tiazídico + } \\
\text { antagonista dos } \\
\text { receptores da } \\
\text { angiotensina }\end{array}$ & $\begin{array}{c}\text { diurérico tiazídico + } \\
\text { antagonista dos } \\
\text { receptores da } \\
\text { angiotensina }\end{array}$ \\
\hline E & betabloqueador & betabloqueador & $\begin{array}{l}\text { bloqueador dos } \\
\text { canais de Cálcio }\end{array}$ & $\begin{array}{l}\text { bloqueador dos } \\
\text { canais de Cálcio }\end{array}$ \\
\hline $\mathrm{F}$ & $\begin{array}{l}\text { bloqueador dos } \\
\text { canais de Cálcio }\end{array}$ & $\begin{array}{l}\text { inibidor da enzima } \\
\text { conversora de } \\
\text { angiotensina }\end{array}$ & betabloqueador & betabloqueador \\
\hline G & $\begin{array}{l}\text { bloqueador dos } \\
\text { canais de Cálcio }\end{array}$ & $\begin{array}{l}\text { bloqueador dos } \\
\text { canais de Cálcio }\end{array}$ & $\begin{array}{c}\text { diurérico tiazídico + } \\
\text { bloqueador dos } \\
\text { canais de Cálcio }\end{array}$ & $\begin{array}{c}\text { diurérico tiazídico + } \\
\text { bloqueador dos } \\
\text { canais de Cálcio }\end{array}$ \\
\hline
\end{tabular}

NOTA: MED (medicação em uso) PRE (pré-operatório) 3M (3 meses) 6M (6 meses) 12M (12 meses) 
O comportamento da pressão arterial nos 5 pacientes em uso de medicação anti-hipertensiva não foi diferente dos outros 2 pacientes. Após 12 meses todos permaneceram hipertensos e os não hipertensos não desenvolveram hipertensão após a cirurgia. Os dados brutos de descenso de todos os pacientes estão nos anexos E e F. 


\section{DISCUSSÃO}

O presente estudo observou um aumento do descenso sistólico e diastólico após a paratireoidectomia em pacientes com HPTP esporádico nas medidas de 6 e 12 meses após a operação.

Os resultados observados na coorte confirmam achados prévios de alta prevalência de hipertensão e descenso atenuado em pacientes com HPTP ${ }^{38}{ }^{40}$ Comparados a outros estudos com MAPA, os resultados aqui descritos são semelhantes aos achados de Petramala et al. de melhora do descenso após paratireoidectomia, com a diferença que no atual estudo observou-se o benefício já aos 6 meses, e, por outro lado, não se observou melhora da pressão arterial sistêmica ${ }^{40}$

Os achados discordam do estudo de Rydberg et al. ${ }^{38}$, que observaram aumento da pressão arterial entre pacientes hipertensos. Ainda que não se tenha analisado separadamente os pacientes hipertensos, eles foram a maioria (72\%), e a pressão arterial sistêmica do grupo não aumentou após a operação.

Ainda em relação às pressões arteriais globais, não se observou diferença ao longo dos 12 meses. Esses achados são consistentes com o recentemente reportado por Concistrè et al. ${ }^{43}$. Por outro lado, eles contrastam com os publicados por Broulik et al..$^{39}$ Heyliger et al. ${ }^{35}$, Petramala et al. ${ }^{40} \mathrm{e}$ Norenstedt et al. ${ }^{41}$ que observaram melhora da pressão arterial sistêmica, sendo os dois últimos com MAPA. Apesar da casuística pequena, o intervalo de confiança dessas variáveis na presente avaliação também foi pequeno. Isso sugere que mesmo com o aumento do número de casos, a conclusão 
deva ser a mesma. Assim, fazem-se necessários mais estudos, com outras metodologias a fim de esclarecer a questão.

O aumento na proporção de pacientes com descenso presente, ainda que não tenha sido estatisticamente significante, indica que alguns indivíduos mudaram de portadores de descenso atenuado para descenso presente, com possível redução de seu risco cardiovascular.

Os presentes resultados sugerem a reversibilidade de um fator de risco cardiovascular associado ao HPTP (o descenso noturno atenuado) após paratireoidectomia. Há quem sugira que as manifestações cardiovasculares do HPTP ocorrem apenas em casos avançados, e não em casos leves ${ }^{18,48}$. Nesse sentido o fato de pacientes aqui analisados terem doença bem estabelecida poderia reduzir a relevância deste achado, no contexto da apresentação moderna da doença. Há, entretanto, evidência de manifestações cardiovasculares em HPTP assintomático, como síndrome metabólica, hipertensão e descenso atenuado, bem como de melhora após paratireoidectomia ${ }^{40}$ Há também indícios de que as manifestações ocorrem de forma gradual com a progressão da doença, e de correlação da pressão sistólica noturna com níveis de $\mathrm{PTH}^{42,49}$. Assim, a demonstração de reversibilidade de um efeito cardiovascular do HPTP, ainda que em doença estabelecida, além de ser um dado que suporta a causalidade da associação, pode indicar que pacientes com formas leves da doença terriam benefícios análogos, entretanto sutis, de acordo com a gravidade do HPTP.

Uma grande limitação do estudo é o grande número de pacientes que não foram aderentes ao protocolo e não realizaram a MAPA nos períodos estabelecidos. Como a grande maioria desses pacientes realizou seus exames 
laboratoriais regularmente nos mesmos momentos em que deveria fazer uma MAPA, pode-se supor que isso foi relacionado com a desgastante rotina necessária para fazê-lo. Há uma série de fatores que podem tornar o exame desconfortável. O deslocamento até o hospital para instalar o monitor e retirálo no dia seguinte pode levar várias horas. Além disso, o uso do aparelho pode atrapalhar atividades cotidianas, trabalho e sono. Em geral, os pacientes toleram o desconforto do exame porque acreditam que ele fornecerá informações relevantes para seu tratamento ${ }^{50,51}$, o que não ocorreu no presente estudo. Em uma tentativa recente de determinar o momento ideal para a repetição da MAPA, com pacientes altamente aderentes, $12 \%$ dos pacientes abandonaram o estudo a cada MAPA repetida ${ }^{52}$.

A inclusão de pacientes hipertensos em uso de medicação e alguns deles trocando de medicação ou até a posologia ao longo da observação também é limitação deste estudo. Entretanto a restrição a pacientes não hipertensos reduziria o universo de possíveis participantes de pesquisa. Além disso, um estudo com tal restrição teria uma população muito distinta da realidade clínica, uma vez que se trata de doença de diagnóstico, na maior parte dos casos, da sexta década de vida em diante, como observado na presente casuística.

Outra limitação é a natureza observacional do estudo, de modo que os clínicos responsáveis pelo cuidado do paciente estiveram livres para mudar a medicação anti-hipertesiva durante o estudo. Mudanças no tratamento antihipertensivo durante o estudo podem ser consideradas um fator de confusão, apesar de os resultados das MAPAs não estarem disponíveis para aqueles médicos. Pode-se supor que o objetivo dessas mudanças não tenha sido 
melhora do descenso noturno, nem que tenha ocorrido ajustes no horário de tomada das medicações com essa finalidade. Já a restrição da troca de medicação anti-hipertensiva durante o estudo parece inadequada dada a natureza da pesquisa realizada (estudo observacional), além de levantar questões éticas.

No presente estudo observou-se uma piora aguda do descenso da PAS e da PAD, mas sem significância estatística. O intervalo de confiança largo pode sugerir que em uma amostra maior o resultado poderia ser significativo. Entre as hipótese para justiçar tal observação pode-se elencar efeitos agudos da paratireoidectomia, com queda abrupta do PTH e cessação de sua ação vasopressora e efeitos residuais das medicações utilizadas no intra-operatório. Essa relação da piora do descenso agudamente poderia ser melhor explorada com uma amostra maior, controle de fatores da técnica anestésica, hidratação, reposição volêmica, suspensão de medicamentos, por exemplo. A piora aguda na creatinina que foi observada é um fato conhecido, mas pouco entendido e pode estar relacionada a ação vasopressora do $\mathrm{PTH}^{53}$. A alteração da creatinina não alcançou significância no presente estudo, nos 7 casos. Contudo houve alteração significativa da creatinina nos 22 casos (dados não apresentados). De fato, na presente casuística a análise dos pacientes que fizeram a MAPA pré-operatória e internados, pode-se verificar aumento ainda maior da Creatinina (de $0,27 \mathrm{mg} / \mathrm{dL}$, ou um aumento de $30 \%$ em relação ao nível basal do grupo, 0,89md/dL, $p=0,0013)$, correspondente a lesão renal aguda grau $1^{54}$.

O estudo não oferece respostas definitivas à questão da mudança da pressão arterial após a paratireoidectomia. A condução deste estudo permite 
apontar para caminhos promissores para estudos futuros sobre o assunto, que parecem necessários, já que apesar do crescente conjunto de evidências sugerindo o aumento de risco cardiovascular e especificamente o aumento da pressão arterial e do descenso noturno, ainda faltam evidências de benefício para a maior parte dos portadores de HPTP, os assintomáticos. Em decorrência disso estes pacientes seguem sendo acompanhados com observação apenas de parâmetros renais e ósseos.

Pode-se sugerir que a utilização de MAPA em futuros estudos deva ser planejada com parcimônia, pois muitas monitorizações tem grande potencial de prejudicar a aderência. Além disso, deve-se tentar implementar medidas que facilitem a realização do exame e amenizem o desconforto, como por exemplo oferecer serviço de coleta do aparelho após o exame ao invés de retirada exclusivamente no hospital. Tal melhoria seria de grande benefício para a aderência ao exame tanto no âmbito de pesquisa como com finalidade assistencial.

Pode-se recomendar estudo pré e pós operatório com MAPA aos 6 e 12 meses em pacientes portadores de HPTP assintomático que não preenchessem critérios propostos para indicação cirúrgica, com dois braços, Um grupo seria observado e outro operado. O primeiro seria o controle e permitira analisar variações não relacionadas à paratireoidectomia. Contudo se no grupo operado confirmarem-se os presentes achados ou a melhora de outros preditores de risco cardiovascular que estiverem ausentes no grupo controle, pode-se afirmar a necessidade de mudança de paradigma (e de conduta, consequentemente). 
Outro aspecto que deve ser observado em estudos futuros é o controle das variáveis de confusão, como por exemplo o tratamento antihipertensivo medicamentoso. Nos vários estudos disponíveis sobre o tema, em geral há controle da medicação anti-hipertensiva. Petramala et al. ${ }^{40}$ observaram redução significativa do uso de medicação anti-hipertensiva em seu estudo, enquanto Norenstedt et al. observaram que 11 de seus 159 pacientes puderam reduzir ou até mesmo cessar o tratamento antihipertensivo $^{41}$, e Storvall et at. observaram aumento não significativo no número de medicamentos anti-hipertensivos ao fim do acompanhamento de 3,3 anos sendo que o número total de medicamentos aumentou e também pode ter influenciado nos resultados já que drogas prescritas com outra finalidade podem alterar a pressão arterial sistêmica ${ }^{44}$.

Outros estudos abordam a questão mais superficialmente. Rydberg et al., assim como Heyliger et al., e Broulik et al. relataram não ter havido mudança no tratamento anti-hipertensivo dos pacientes hipertensos ${ }^{35,38,39}$, observação que diverge da realidade clínica relatada no presente trabalho, principalmente nos dois últimos casos, onde há grande número de pacientes em tratamento medicamentoso anti-hipertensivo. Já Conscistrè et al. realizaram retirada do tratamento anti-hipertensivo por 14 dias antes da realização da MAPA em seus pacientes, a fim de minimizar a interferência da medicação, tanto antes quanto após a operação. Relatam ainda que, entre os 10 pacientes hipertensos operados, o tratamento anti-hipertensivo estava igual ou reduzido ao fim do seguimento ${ }^{43}$. 
Por outro lado, há autores que não relataram o controle de medicações anti-hipertensivas em seus estudos, o que diminui a validade de seus resultados ${ }^{36,37}$.

Apesar de não ser ético proibir a mudança no tratamento antihipertensivo, devem-se manter os cuidados clínicos com os sujeitos de pesquisa a critério das equipes do hospital e participante da pesquisa, de modo a minimizar as mudanças de medicação e ter clareza das indicações para tal quando for necessário. Dessa forma seria possível esclarecer a questão da melhora da pressão arterial, bem como o momento em que isso ocorre. 


\section{CONCLUSÃO}

Em pacientes com HPTP esporádico submetidos a paratireoidectomia curativa, ocorre melhora do descenso noturno da pressão arterial sistólica e diastólica, observada aos 6 meses após a operação e sustentada até 12 meses. 
Anexo A - Termo de Consentimento Livre e Esclarecido

hospital das clínicas da faculdade de MEdicina da UNIVERSidade de SÃo PAULO-HCFMUSP

TERMO DE CONSENTIMENTO LIVRE E ESCLARECIDO

DADOS DE IDENTIFICAÇÃo DO SUJEITO DA PESQUISA OU RESPONSÁVEL LEGAL

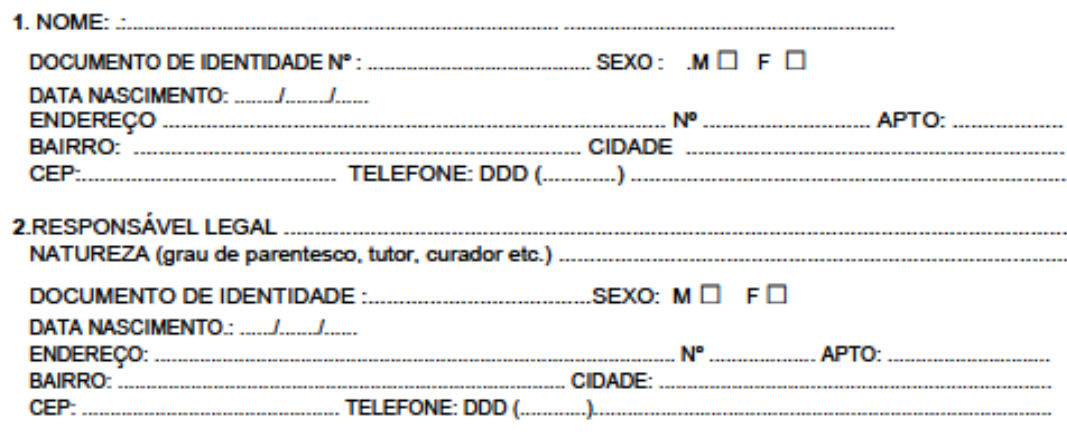

DADOS SOBRE A PESQUISA

1. TITULO DO PROTOCOLO DE PESQUISA: AVALIACÅ̃O DA PRESSÃO ARTERIAL SISTEMMICA ANTES E APÓS PARATIREOIDECTOMIA POR HIPERPARATIREOIDISMO PRIMÁRIO.

2. PESQUISADOR : LEDO MAZZEI MASSONI NETO

CARGOVFUNÇÃO: MÉDICO INSCRIÇÅO CONSELHO REGIONAL N N $^{\circ} 124985$

UNIDADE DO HCFMUSP: DISCIPLINA DE CIRURGIA DE CABEÇA E PESCOÇO

3. AVALIAÇÅO DO RISCO DA PESQUISA:

$\begin{array}{ll}\text { RISCO MINIMO X } & \text { RISCO MÉDIO } \square \\ \text { RISCO BAIXO } \square & \text { RISCO MAIOR } \square\end{array}$

4.DURAÇÅ̄O DA PESQUISA :12 MESES 
HOSPITAL DAS CLÍNICAS DA FACULDADE DE MEDICINA

DA UNIVERSIDADE DE SÃO PAULO-HCFMUSP

1 - Estas informaçöes estäo sendo fornecidas para sua participação voluntária em um estudo. Este estudo visa analisar a variaçäo da pressäo do sangue (pressão arterial) antes e após a cirurgia de remoção de uma ou mais glândulas paratireóides (paratireoidectomia), que é o tratamento recomendado para casos de funcionamento em excesso destas glândulas (hiperparatireoidismo). Esta é a doença que o(a) senhor(a) tem.

2 - Este é um estudo de observaçäo, isto é, nenhum tratamento novo será testado. O tratamento que será feito no(a) senhor(a) é exatamente o mesmo que realizamos em pacientes com a sua condiçäo em nosso hospital. 3 - Relação dos procedimentos rotineiros e como säo realizados: Seräo registrados fatores como idade, peso altura, dados sobre a cirurgia e anestesia (duraçäo, eventos não esperados, medicamentos empregados, quantidade de soro recebida, pressäo arterial durante a cirurgia), diurese de 24 horas (volume de urina eliminado em 24 horas), calciúria (quantidade de cálcio eliminado na urina), monitorização da pressão arterial (medida de pressäo a cada quinze minutos por aparelho semelhante a um medidor de pressäo convencional, que ficará no braço do senhor(a) po 24 horas), alguns exames laboratoriais de sangue além dos que seriam realizados rotineiramente em seu acompanhamento, mas a coleta ("picada") é a mesma da rotina de cuidado. Seräo realizados coleta de sangue por punçäo periférica da veia do antebraço, na véspera da cirurgia, no primeiro e terceiro dias após a cirurgia, no sétimo dia após a cirurgia e novamente um mês, três meses, seis meses e doze meses após a cirurgia; Monitorização da pressão arterial: será monitorizada a pressäo arterial na véspera da cirurgia, primeiro e terceiro dias após a cirurgia, no sétimo dia após a cirurgia e novamente um mès, três meses, seis meses e doze meses após a cirurgia; Exames de urina: A diurese e o cálcio na urina de 24 horas serão medidas no pré-operatório, no primeiro, segundo e terceiro dia pós-operatório, em 6 meses e em 12 meses após a cirurgia.

4 - Os desconfortos e riscos causados pelos procedimentos do estudo säo: o desconforto causado pelo monitor de pressão arterial, e as complicações decorrentes de uma coleta de sangue da veia , que säo raras, suportáveis. facilmente percebidos e tratáveis. As mais frequentes säo: dor, formação de coágulo no local da coleta ou infeç̧ão. 5 -Possiveis benefícios para o(a) senhor(a) da participaçäo neste estudo seräo: detectar hipertensäo arterial previamente desconhecida, controlar adequadamente hipertensäo arterial mal controlada, detectar a partir dos exames de sangue e urina doença dos rins previamente desconhecidas. Além destes possíveis beneficios, a realizaçäo deste estudo permitirá melhor entendimento da doença do senhor(a), o que poderá ajudar pessoas $\infty m$ a sua condiçäo no futuro.

6 - Relação de procedimentos alternativos que possam ser vantajosos, pelos quais o paciente pode optar. Este estudo não testa nenhum novo tratamento, e dessa forma näo há procedimentos alternativos.

7 - Garantia de acesso: em qualquer etapa do estudo, você terá acesso aos profissionais responsáveis pela pesquisa para esclarecimento de eventuais dúvidas. O principal investigador é o Dr Ledo Mazzei Massoni Neto. que pode ser encontrado no endereço Avenida Dr Enéas de Carvalho Aguiar, 455, Disciplina de Cirurgia de Cabeça e Pescoço, $8^{\circ}$ andar. Telefone(s) 11 30696425. Se você tiver alguma consideraçäo ou dúvida sobre a ética da pesquisa, entre em contato com o Comitè de Ética em Pesquisa (CEP) - Rua Ovídio Pires de Campos, $225-5^{\circ}$ andar - tel: 3069-6442 ramais 16, 17, 18 ou 20, FAX-3069-6442 ramal 26 - E-mail: cappesq@henet.usp.br 8 -É garantida a líberdade da retirada de consentimento a qualquer momento e deixar de participar do estudo. sem qualquer prejuízo à continuidade de seu tratamento na Instituição; 
09 - Direito de confidencialidade - As informaçöes obtidas seräo analisadas em conjunto com outros pacientes. näo sendo divulgado a identificação de nenhum paciente;

10 - Direito de ser mantido atualizado sobre os resultados parciais das pesquisas, quando em estudos abertos, ou de resultados que sejam do conhecimento dos pesquisadores;

11 - Despesas e compensações: não há despesas pessoais para o participante em qualquer fase do estudo. incluindo exames e consultas. Também não há compensaçäo financeira relacionada à sua participaçäo.

12 - Compromisso do pesquisador de utilizar os dados e o material coletado somente para esta pesquisa.

Acredito ter sido suficientemente informado a respeito das informações que li ou que foram lidas para mim, descrevendo o estudo" AVALIAÇÃO DA PRESSÃO ARTERIAL SISTĖMICA ANTES E APÓS PARATIREOIDECTOMIA POR HIPERPARATIREOIDISMO PRIMÁRIO

Eu discuti com o Dr. Ledo Mazzei Massoni Neto ou com outros médicos participantes do estudo sobre a minha decisão em participar nesse estudo. Ficaram claros para mim quais são os propósitos do estudo, os procedimentos a serem realizados, seus desconfortos e riscos, as garantias de confidencialidade e de esclarecimentos permanentes. Ficou claro também que minha participação é isenta de despesas e que tenho garantia do acesso a tratamento hospitalar quando necessário. Concordo voluntariamente em participar deste estudo e poderei retirar o meu consentimento a qualquer momento, antes ou durante o mesmo, sem penalidades ou prejuizo ou perda de qualquer benefício que eu possa ter adquirido, ou no meu atendimento neste Serviço.

Assinatura do paciente/representante legal Data

11

para casos de pacientes menores de 18 anos, analfabetos, semi-analfabetos ou portadores de deficiência auditiva ou visual.

\section{(Somente para o responsável do projeto)}

Declaro que obtive de forma apropriada e voluntária o Consentimento Livre e Esclarecido deste paciente ou representante legal para a participação neste estudo. 
Anexo B - Planejamento das MAPAS

PROTOCOLO DE MAPA PARA PACIENTES COM HIPERPARATIREOIDISMO

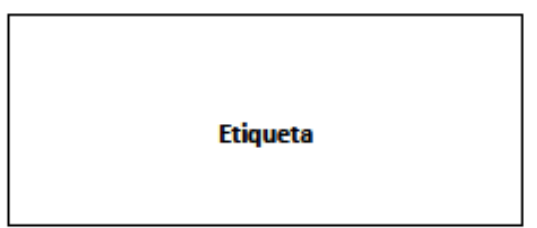

№ PROTOCOLO:

DATA INCLUSÃO:

\section{DATA DA CIRURGIA:}

1. MAPA PRÉ-OP, AMBULATORIAL, 5 a F

2. CIRURGIA 2a $\mathbf{F}$

3. MAPA - 10 PO, INTERNADO, 3a $F$

4. MAPA - 3O PO, INTERNADO, 5 a $F$

5. MAPA - 9o PO, AMBULATORIAL, 4a F

\begin{tabular}{|c|c|c|c|c|}
\hline & PRÉ-OP & 10 PO & 3o PO & 9o PO \\
\hline DIA & 5äF & 3aF & 5aF & 4aF \\
\hline REGIME & AMBULATORIAL & INTERNADO & INTERNADO & AMBULATORIAL \\
\hline HORÁRIO & 10H & 10H & 10H & 10H \\
\hline DATA: & & & & \\
\hline FEITO (CHECAR) & & & & \\
\hline
\end{tabular}

1. MAPA - 1 MÊS PO, AMBULATORIAL

2. MAPA - 3 MESES PO, AMBULATORIAL

3. MAPA - 6 MESES PO, AMBULATORIAL

4. MAPA COM 1 ANO DE PÓS OPERATÓRIO, AMBULATORIAL

\begin{tabular}{|c|c|c|c|c|}
\hline & 1 MÊS & 3 MESES & 6 MESES & 1 ANO \\
\hline REGIME & AMBULATORIAL & AMBULATORIAL & AMBULATORIAL & AMBULATORIAL \\
\hline HORÁRIO & $10 \mathrm{H}$ & $10 \mathrm{H}$ & $10 \mathrm{H}$ & $10 \mathrm{H}$ \\
\hline DATA: & & & & \\
\hline FEITO (CHECAR) & & & & \\
\hline
\end{tabular}

1 VIA - AMBULATÓRIO MAPA

2* VIA-PACIENTE

$3^{*}$ VIA-CABEÇA E PESCOÇO 


\section{Anexo C - Aprovação pelo Comitê de Ética em Pesquisa}

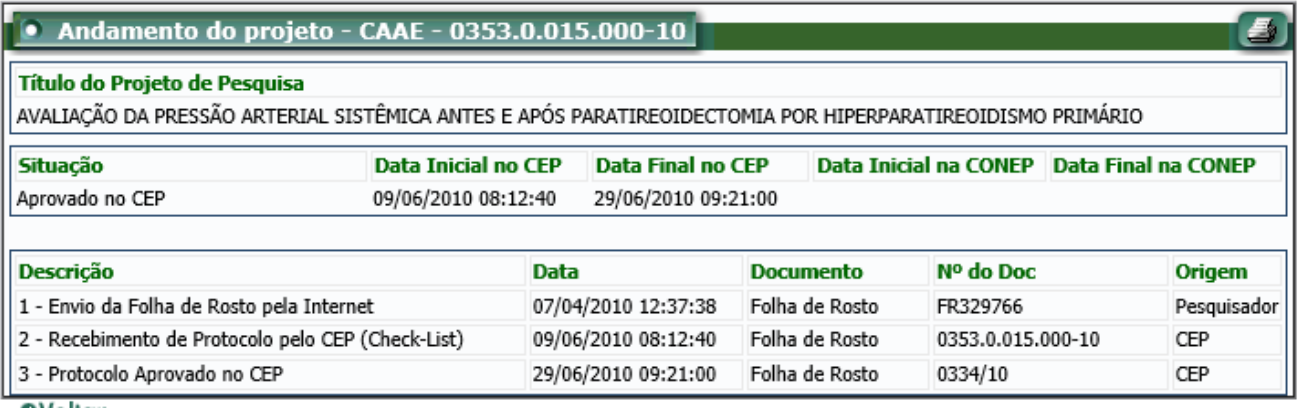




\section{Anexo D - Alteração da finalidade acadêmica para doutorado}

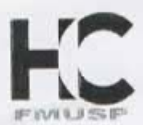

$N^{\circ}$ Protocolo: $0334 / 10$

Título: AVALIAÇÃO DA PRESSÃO ARTERIALEMICA ANTES E APÓSPORPRIMÁRIO

Pesquisador Responsável: Fabio Luiz de Menezes Montnegro

Pesquisador Executante: Ledo Mazzei Massoni Neto

Disciplina: Cirurgia de Cabeça e Pescoço

Departamento: CIRURGIA

A Comissão de Ética para Análise de Projetos de Pesquisa CAPpesa da Diretoria Clinica do Hospital das Clinicas da Faculdade de Medicina da Universidade de São Paulo, APROVOU / TOMOU CIÊNCIA na sessão datada de 09/04/2014, do(s) documento(s) abaixo mencionado(s):

- Carta datada de 26/03/2014 - Relatório Parcial e solicitaçāo de alteração de finalidade acadêmica.

Em conformidade com a Resoluçăo CNS $n^{\circ} 466 / 12$ - cabe ao pesquisador: a) desenvolver o projeto conforme delineado: b) elaborar $e$ apresentar relatórios parciais e final: c)apresentar dados solicitados pelo CEP, a qualquer momento; d) manter em arquivo sob sua guarda, por 5 anos da pesquisa, contendo fichas individuais e todos os demais documentos recomendados pelo CEP: e) encaminhar os resultados para publicação, com os devidos créditos aos pesquisadores associados e ao pessoal técnico participante do projeto: f) justificar perante ao CEP interrupção do projeło ou a não publicação dos resultados.

CAPPesq, 09 de Abril de 2014

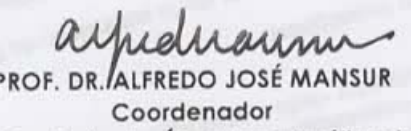

Comissão de Ética para Análise de

Projetos de Pesquisa - CAPPesq

Pua Dr Ovidio Pires de Campos, 225 - Prédio da Administraçáo - $5^{\circ}$ andar - CEP 05403-010 - Sấo Paulo - SP 5511 2661-7585-55 112661.6442 ramais: 16, 17, 18 | marcia.carvalho@hc.fm. usp.br 
Anexo E - Dados demográficos e bioquímicos dos 22 pacientes incluídos

\begin{tabular}{|c|c|c|c|c|c|c|}
\hline CASO & $\begin{array}{r}\text { IDADE } \\
\text { (anos) }\end{array}$ & $\begin{array}{c}\text { PTH PRE } \\
(\mathrm{pg} / \mathrm{mL})\end{array}$ & $\begin{array}{c}\text { CaT PRE } \\
\text { (mg/dL) }\end{array}$ & $\begin{array}{l}\text { Cai PRE } \\
\text { (mg/dL) }\end{array}$ & $\begin{array}{l}\text { P PRE } \\
\text { (mg/dL) }\end{array}$ & $\begin{array}{l}\text { Cr PRE } \\
(\mathrm{mg} / \mathrm{dL})\end{array}$ \\
\hline$A$ & 73 & 108,00 & 10,40 & 5,20 & 3,20 & 1,05 \\
\hline$B$ & 68 & 145,00 & 9,30 & 5,41 & 2,60 & 1,20 \\
\hline C & 52 & 100,00 & 9,70 & 5,40 & 3,40 & 0,94 \\
\hline $\mathrm{D}$ & 64 & 226,00 & 11,80 & 6,40 & 2,40 & 0,82 \\
\hline$E$ & 62 & 121,00 & 9,40 & 5,40 & 3,70 & 0,88 \\
\hline$F$ & 64 & 110,00 & 9,60 & 5,20 & 3,50 & 1,18 \\
\hline$G$ & 77 & 314,00 & 12,20 & 7,50 & 2,00 & 0,92 \\
\hline $\mathrm{H}$ & 55 & 164,00 & 11,80 & 6,80 & 2,30 & 0,68 \\
\hline I & 60 & 251,00 & 10,20 & 5,40 & 3,30 & 1,52 \\
\hline $\mathrm{J}$ & 57 & 297,00 & 9,90 & 5,30 & 2,30 & 0,67 \\
\hline $\mathrm{K}$ & 46 & 176,00 & 9,60 & 5,70 & 2,60 & 0,59 \\
\hline $\mathrm{L}$ & 57 & 212,00 & 11,40 & 6,70 & 1,80 & 0,76 \\
\hline$M$ & 80 & 119,00 & 11,20 & 6,30 & 2,80 & 0,91 \\
\hline $\mathrm{N}$ & 54 & 151,00 & 10,90 & 5,70 & 2,70 & 0,91 \\
\hline 0 & 52 & 204,00 & 10,40 & 5,70 & 3,30 & 0,62 \\
\hline$P$ & 59 & 123,00 & 10,20 & 5,40 & 3,10 & 0,84 \\
\hline$Q$ & 45 & 732,00 & 12,50 & 7,90 & 1,50 & 0,76 \\
\hline $\mathrm{R}$ & 58 & 75,00 & 10,50 & 5,50 & 3,00 & 0,90 \\
\hline$S$ & 78 & 94,00 & 10,40 & 5,17 & 3,60 & 0,77 \\
\hline $\mathrm{T}$ & 59 & 295,00 & 12,00 & 6,20 & 2,60 & 1,02 \\
\hline$U$ & 55 & 208,00 & 11,80 & 6,30 & 3,20 & 0,64 \\
\hline V & 38 & 492,00 & 12,60 & 7,80 & 2,40 & 0,99 \\
\hline
\end{tabular}

NOTA: PTH (paratormônio sérico), CaT (cálcio total sérico), Cai (cálcio iônico sérico), $\mathrm{P}$ (fósforo sérico), $\mathrm{Cr}$ (creatinina sérica), $\mathrm{mg} / \mathrm{dL}$ (miligramas por decilitro), $\mathrm{pg} / \mathrm{mL}$ (picogramas por mililitro) 
Anexo F - Descenso noturno da pressão arterial sistêmica dos 22 casos (\%)

\begin{tabular}{|c|c|c|c|c|c|c|c|c|c|c|}
\hline CASO & $\begin{array}{c}\text { DESC } \\
\text { PAS } \\
\text { PRE }\end{array}$ & $\begin{array}{c}\text { DESC } \\
\text { PAD } \\
\text { PRE }\end{array}$ & $\begin{array}{c}\text { DESC } \\
\text { PAS } \\
\text { 3PO }\end{array}$ & $\begin{array}{c}\text { DESC } \\
\text { PAD } \\
3 P O\end{array}$ & $\begin{array}{c}\text { DESC } \\
\text { PAS } \\
3 \mathrm{M}\end{array}$ & $\begin{array}{c}\text { DESC } \\
\text { PAD } \\
3 M\end{array}$ & $\begin{array}{c}\text { DESC } \\
\text { PAS } \\
6 M\end{array}$ & $\begin{array}{c}\text { DESC } \\
\text { PAD } \\
6 \mathrm{M}\end{array}$ & $\begin{array}{c}\text { DESC } \\
\text { PAS } \\
12 \mathrm{M}\end{array}$ & $\begin{array}{c}\text { DESC } \\
\text { PAD } \\
12 \mathrm{M}\end{array}$ \\
\hline$A$ & $-3,45$ & 2,90 & 8,33 & 16,44 & 7,41 & 14,93 & 6,96 & 16,90 & 1,77 & 11,59 \\
\hline B & $-3,23$ & $-1,30$ & $-2,99$ & $-2,56$ & 5,56 & 6,41 & 4,62 & 6,17 & 3,85 & 9,41 \\
\hline C & 13,33 & 13,51 & 10,74 & 8,22 & 14,29 & 21,62 & 14,29 & 21,92 & 21,43 & 30,77 \\
\hline$D$ & 8,59 & 8,45 & 0,79 & 3,13 & 8,89 & 13,16 & 13,24 & 14,86 & 10,00 & 12,66 \\
\hline$E$ & $-2,65$ & $-1,52$ & 20,18 & $\begin{array}{c}- \\
19,40\end{array}$ & & & 5,26 & $-1,47$ & 10,08 & 9,33 \\
\hline$F$ & 4,11 & 7,87 & 8,39 & 17,02 & 0,62 & 2,02 & 12,58 & 8,51 & 4,73 & 2,02 \\
\hline $\mathrm{G}$ & 13,24 & 19,48 & 3,62 & 5,33 & 17,14 & 23,75 & 17,99 & 26,44 & 21,90 & 27,06 \\
\hline $\mathrm{H}$ & 6,98 & 21,84 & 0,78 & 5,13 & 15,91 & 26,88 & 2,56 & 21,95 & 15,15 & 24,42 \\
\hline I & & & 4,90 & 4,26 & 11,92 & 17,00 & 8,00 & 13,27 & 6,80 & 15,96 \\
\hline $\mathrm{J}$ & 1,46 & 1,14 & 8,97 & 7,95 & 7,69 & 5,62 & 4,44 & 1,20 & & \\
\hline K & 13,56 & 20,00 & 6,92 & 13,10 & 2,36 & 6,67 & 11,48 & 24,68 & & \\
\hline$L$ & 16,54 & 19,32 & 6,90 & 8,51 & 12,80 & 18,29 & 11,02 & 19,28 & & \\
\hline$M$ & 7,63 & 10,81 & $-6,82$ & 0,00 & $-5,69$ & $-2,99$ & & & & \\
\hline $\mathrm{N}$ & 4,88 & 6,58 & 5,79 & 6,41 & & & & & & \\
\hline 0 & 14,19 & 12,87 & 12,31 & 12,79 & & & & & & \\
\hline$P$ & 2,42 & 15,66 & & & $-6,20$ & 6,17 & & & & \\
\hline Q & 7,75 & 10,87 & 15,79 & 19,28 & & & & & & \\
\hline $\mathrm{R}$ & 4,11 & 11,49 & 5,52 & 12,94 & & & & & & \\
\hline$S$ & 7,91 & 10,47 & 1,72 & 6,06 & & & & & & \\
\hline $\mathrm{T}$ & 5,50 & 22,22 & & & & & & & & \\
\hline U & 21,83 & 26,37 & & & & & & & & \\
\hline V & 16,91 & 21,33 & & & & & & & & \\
\hline
\end{tabular}

NOTA: DESC (descenso noturno) PRE (pré-operatório) 3PO (terceiro pós operatório), $3 \mathrm{M}$ (3 meses), 6M (6 meses) 12M (12 meses) PAS (pressão arterial sistólica), PAD (pressão arterial diastólica) 


\section{REFERÊNCIAS BIBLIOGRÁFICAS}

1. Marcocci C, Cetani F. Clinical practice. Primary hyperparathyroidism. N Engl J Med. 2011 Dec 22;365(25):2389-97.

2. Fraser WD. Hyperparathyroidism. The Lancet. Elsevier Ltd; 2009 Jul 11;374(9684):145-58.

3. Melton LJ. The epidemiology of primary hyperparathyroidism in North America. J Bone Miner Res. 2002 Nov;17 Suppl 2:N12-7.

4. Yu N, Donnan PT, Murphy MJ, Leese GP. Epidemiology of primary hyperparathyroidism in Tayside, Scotland, UK. Clin Endocrinol. 2009 Sep 7;71(4):485-93.

5. Marcocci C, Saponaro F. Epidemiology, pathogenesis of primary hyperparathyroidism: Current data. Annales d'Endocrinologie. 2015 May;76(2):113-5.

6. Griebeler ML, Kearns AE, Ryu E, Hathcock MA, Melton LJI, Wermers RA. Secular trends in the incidence of primary hyperparathyroidism over five decades (1965-2010). Bone. 2015 Apr;73:1-7.

7. Bilezikian JP, Bandeira L, Khan A, Cusano NE. Seminar Hyperparathyroidism. The Lancet. Elsevier Ltd; 2017 Sep 14;391(10116):1-11.

8. Ohe MN, Santos RO, Barros ER, Lage A, Kunii IS, Abrahão M, et al. Changes in clinical and laboratory findings at the time of diagnosis of primary hyperparathyroidism in a University Hospital in São Paulo from 1985 to 2002. Braz J Med Biol Res. 2005 Sep;38(9):1383-7.

9. Eufrazino C, Veras A, Bandeira F. Epidemiology of Primary Hyperparathyroidism and its Non-classical Manifestations in the City of Recife, Brazil. Clin Med Insights Endocrinol Diabetes. 2013;6:6974.

10. Silverberg SJ, Bilezikian JP. "Incipient” Primary Hyperparathyroidism: A 'Forme Fruste' of an Old Disease. The Journal of Clinical Endocrinology \& Metabolism. 2003 Nov;88(11):5348-52.

11. Cusano NE, Silverberg SJ, Bilezikian JP. Normocalcemic primary hyperparathyroidism. J Clin Densitom. 2013 Jan;16(1):33-9.

12. Silverberg SJ, Shane E, Jacobs TP, Siris E, Bilezikian JP. A 10-year prospective study of primary hyperparathyroidism with or without parathyroid surgery. N Engl J Med. 1999 Oct 21;341(17):1249-55. 
13. Rubin MR, Bilezikian JP, McMahon DJ, Jacobs T, Shane E, Siris E, et al. The Natural History of Primary Hyperparathyroidism with or without Parathyroid Surgery after 15 Years. The Journal of Clinical Endocrinology \& Metabolism. 2008 Sep;93(9):3462-70.

14. Bilezikian JP, Brandi ML, Eastell R, Silverberg SJ, Udelsman R, Marcocci C, et al. Guidelines for the Management of Asymptomatic Primary Hyperparathyroidism: Summary Statement from the Fourth International Workshop. The Journal of Clinical Endocrinology \& Metabolism. 2014 Oct;99(10):3561-9.

15. Bilezikian JP, Cusano NE, Khan AA, Liu J-M, Marcocci C, Bandeira F. Primary hyperparathyroidism. Nat Rev Dis Primers. 2016 May 19;2:16033-8.

16. Khan AA, Hanley DA, Rizzoli R, Bollerslev J, Young JEM, Rejnmark $\mathrm{L}$, et al. Primary hyperparathyroidism: review and recommendations on evaluation, diagnosis, and management. A Canadian and international consensus. Osteoporos Int. Osteoporosis International; 2016 Dec 18;28(1):1-19.

17. Bilezikian J. Primary Hyperparathyroidism. Endocrine Practice. 2012 Sep;18(5):781-90.

18. Walker MD, Rubin M, Silverberg SJ. Nontraditional manifestations of primary hyperparathyroidism. J Clin Densitom. 2013 Jan;16(1):40-7.

19. Hellströum J, Birke G, Edvall CA. HYPERTENSION IN HYPERPARATHYROIDISM1. BJU International. Blackwell Publishing Ltd; 1958 Mar 1;30(1):13-24.

20. Rosenthal FD, Roy S. Hypertension and hyperparathyroidism. $\mathrm{Br}$ Med J. BMJ Publishing Group; 1972 Nov 18;4(5837):396-7.

21. Chowdhury SD, Gray JG. Renal function and hypertension in primary hyperparathyroidism. Br J Surg. 1973 Jan;60(1):53-6.

22. Jones DB, Jones JH, Lloyd HJ, Lucas PA, Wilkins WE, Walker DA. Changes in blood pressure and renal function after parathyroidectomy in primary hyperparathyroidism. Postgraduate Medical Journal. BMJ Publishing Group; 1983 Jun;59(692):350-3.

23. Ronni-Sivula H. Causes of death in patients previously operated on for primary hyperparathyroidism. Ann Chir Gynaecol. 1985;74(1):138.

24. Ljunghall S, Jakobsson S, Joborn C, Palmér M, Rastad J, Akerstrom G. Longitudinal studies of mild primary hyperparathyroidism. J Bone Miner Res. John Wiley and Sons and The American Society for Bone and Mineral Research (ASBMR); 1991 Oct;6 Suppl 2(S2):S111-6-discussionS121-4. 
25. Silverberg SJ. Non-classical target organs in primary hyperparathyroidism. J Bone Miner Res. 2002 Nov;17 Suppl 2:N117-25.

26. Vestergaard PEA. Cardiovascular Events before and after Surgery for Primary Hyperparathyroidism. 2003 Jan 27;:1-7.

27. Vaidya A, Curhan GC, Paik JM, Kronenberg H, Taylor EN. Hypertension, Antihypertensive Medications, and Risk of Incident Primary Hyperparathyroidism. The Journal of Clinical Endocrinology \& Metabolism. 2015 Jun;100(6):2396-404.

28. Kalla A, Krishnamoorthy P, Gopalakrishnan A, Garg J, Patel NC, Figueredo VM. Primary hyperparathyroidism predicts hypertension: Results from the National Inpatient Sample. International Journal of Cardiology. Elsevier Ireland Ltd; 2016 Nov 11;227:1-3.

29. Pickering TG, Shimbo D, Haas D. Ambulatory blood-pressure monitoring. N Engl J Med. 2006 Jun 1;354(22):2368-74.

30. Staessen JA, Asmar R, De Buyzere M, Imai Y, Parati G, Shimada K, et al. Task Force II: Blood pressure measurement and cardiovacular outcome. Blood Pressure Monitoring. 2001 Dec;6(6):355-70.

31. Ohkubo T, Hozawa A, Yamaguchi J, Kikuya M, Ohmori K, Michimata $\mathrm{M}$, et al. Prognostic significance of the nocturnal decline in blood pressure in individuals with and without high 24-h blood pressure: the Ohasama study. Journal of Hypertension. 2002 Nov;20(11):2183-9.

32. Salles GF, Reboldi G, Fagard RH, Cardoso CRL, Pierdomenico SD, Verdecchia P, et al. Prognostic Effect of the Nocturnal Blood Pressure Fall in Hypertensive Patients: The Ambulatory Blood Pressure Collaboration in Patients With Hypertension (ABC-H) Meta-Analysis. Hypertension. American Heart Association, Inc; 2016 Apr;67(4):693-700.

33. Nuzzo V, Tauchmanovà L, Fonderico F, Trotta R, Fittipaldi MR, Fontana $\mathrm{D}$, et al. Increased intima-media thickness of the carotid artery wall, normal blood pressure profile and normal left ventricular mass in subjects with primary hyperparathyroidism. European Journal of Endocrinology. 2002 Oct;147(4):453-9.

34. Letizia C, Ferrari P, Cotesta D, Caliumi C, Cianci R, Cerci S, et al. Ambulatory monitoring of blood pressure (AMBP) in patients with primary hyperparathyroidism. J Hum Hypertens. 2005 Jul 7;19(11):901-6.

35. Heyliger A, Tangpricha V, Weber C, Sharma J. Parathyroidectomy decreases systolic and diastolic blood pressure in hypertensive patients with primary hyperparathyroidism. Surgery. Mosby, Inc; 
2009 Dec 1;146(6):1042-7.

36. Bollerslev J, Rosen T, Mollerup CL, Nordenström J, Baranowski M, Franco C, et al. Effect of Surgery on Cardiovascular Risk Factors in Mild Primary Hyperparathyroidism. The Journal of Clinical Endocrinology \& Metabolism. 2009 Jul;94(7):2255-61.

37. Feldstein CA, Akopian M, Pietrobelli D, Olivieri A, Garrido D. LongTerm Effects of Parathyroidectomy on Hypertension Prevalence and Circadian Blood Pressure Profile in Primary Hyperparathyroidism. Clinical and Experimental Hypertension. 2010 May 26;32(3):154-8.

38. Rydberg E, Birgander M, Bondeson A-G, Bondeson L, Willenheimer $R$. Effect of successful parathyroidectomy on 24-hour ambulatory blood pressure in patients with primary hyperparathyroidism. International Journal of Cardiology. Elsevier Ireland Ltd; 2010 Jun 25;142(1):15-21.

39. Broulik PD, Brouliková A, Adámek S, Libanský P, Tvrdoň J, Broulikova K, et al. Improvement of Hypertension after Parathyroidectomy of Patients Suffering from Primary Hyperparathyroidism. International Journal of Endocrinology. 2011;2011(9):1-6.

40. Petramala P, Formicuccia FM, Zinnamosca Z, Marinelli M, Cilenti C, Colangelo C, et al. Arterial Hypertension, Metabolic Syndrome and Subclinical Cardiovascular Organ Damage in Patients with Asymptomatic Primary Hyperparathyroidism before and after Parathyroidectomy: Preliminary Results. International Journal of Endocrinology. 2012;2012(5):408295-10.

41. Norenstedt S, Pernow Y, Brismar K, Sääf M, Ekip A, Granath F, et al. Primary hyperparathyroidism and metabolic risk factors, impact of parathyroidectomy and vitamin $\mathrm{D}$ supplementation, and results of a randomized double-blind study. European Journal of Endocrinology. European Society of Endocrinology; 2013 Dec;169(6):795-804.

42. Nilsson I-L, Norenstedt S, Granath F, Zedenius J, Pernow Y, Larsson TE. FGF23, metabolic risk factors, and blood pressure in patients with primary hyperparathyroidism undergoing parathyroid adenomectomy. Surgery. 2016 Jan;159(1):211-7.

43. Concistrè A, Grillo A, La Torre G, Carretta R, Fabris B, Petramala L, et al. Ambulatory blood pressure monitoring-derived short-term blood pressure variability in primary hyperparathyroidism. Endocrine. Springer US; 2017 Jul 11;365(2):1-8.

44. Storvall S, Ryhänen E, Heiskanen I, Sintonen H, Roine R, SchalinJäntti C. Surgery Significantly Improves Neurocognition, Sleep, and Blood Pressure in Primary Hyperparathyroidism: A 3-Year 
Prospective Follow-Up Study. Horm Metab Res. 2017 Oct 5;49(10):772-7.

45. Carneiro DM, Solorzano CC, Nader MC, Ramirez M, Irvin GL III. Comparison of intraoperative iPTH assay (QPTH) criteria in guiding parathyroidectomy: Which criterion is the most accurate? Surgery. 2003 Dec;134(6):973-9.

46. O'Brien E, Mee F, Atkins N, O'Malley K. Accuracy of the SpaceLabs 90207 determined by the British Hypertension Society protocol. Journal of Hypertension. 1991 Jun;9(6):573-4.

47. Grupo de Trabalho MAPA, Grupo de Trabalho MRPA. [V Brazilian guidelines for ambulatory monitoring of arterial pressure and III Brazilian guidelines for home monitoring of blood pressure]. Vol. 33, Jornal brasileiro de nefrologia : 'orgao oficial de Sociedades Brasileira e Latino-Americana de Nefrologia. 2011. pp. 365-88.

48. Wermers RA, Khosla S, Atkinson EJ, Grant CS, Hodgson SF, O'Fallon WM, et al. Survival after the diagnosis of hyperparathyroidism: a population-based study. Am J Med. 1998 Feb;104(2):115-22.

49. Hedbäck G, Oden A. Increased risk of death from primary hyperparathyroidism--an update. Eur J Clin Invest. 1998 Apr;28(4):271-6.

50. Little P, Barnett J, Barnsley L, Marjoram J, Fitzgerald-Barron A, Mant D. Comparison of acceptability of and preferences for different methods of measuring blood pressure in primary care. BMJ. 2002 Aug 3;325(7358):258-9.

51. Ernst ME, Bergus GR. Favorable patient acceptance of ambulatory blood pressure monitoring in a primary care setting in the United States: a cross-sectional survey. BMC Fam Pract. 2003 Oct 8;4(1):1065-6.

52. Muxfeldt ES, Fiszman R, de Souza F, Viegas B, 2012. Appropriate time interval to repeat ambulatory blood pressure monitoring in patients with white-coat resistant hypertension. Am Heart Assoc. 2012 Jan 18;59(2):384-9.

53. Montenegro FL de M, Martin RM, Corrêa PHS. Renal failure after surgery for primary hyperparathyroidism: is acute reduction of parathyroid function a risk factor? Clinics. 2009;64(4):1-4.

54. Khwaja A. KDIGO Clinical Practice Guidelines for Acute Kidney Injury. Nephron. 2012 Oct 1;120(4):c179-84. 